\title{
Planetary period oscillations in Saturn's magnetosphere: Evidence in magnetic field phase data for rotational modulation of Saturn kilometric radiation emissions
}

\author{
D. J. Andrews, ${ }^{1}$ B. Cecconi, ${ }^{2}$ S. W. H. Cowley, ${ }^{1}$ M. K. Dougherty, ${ }^{3}$ L. Lamy, ${ }^{2}$ G. Provan, ${ }^{1}$ \\ and P. Zarka ${ }^{2}$
}

Received 9 March 2011; revised 27 May 2011; accepted 13 June 2011; published 10 September 2011.

[1] Initial Voyager observations of Saturn kilometric radiation (SKR) indicated that the modulations in emitted power near the $\sim 11 \mathrm{~h}$ planetary rotation period are "strobe like," varying with a phase independent of observer position, while subsequent Cassini studies of related oscillations in the magnetospheric magnetic field and plasma parameters have shown that these rotate around the planet with a period close to the SKR period. However, analysis of magnetic oscillation data over the interval 2004-2010 reveals the presence of variable secular drifts between the phases of the dominant southern period magnetic oscillations and SKR modulations, which become very marked after Cassini apoapsis moved for the first time into the postdusk sector in mid-2009. Here we use a simple theoretical model to show that such phase drifts arise if the SKR modulation phase also rotates around the auroral oval, combined with a highly restricted view of the SKR sources by the spacecraft due to the conical beaming of the emissions. Strobe-like behavior then occurs in the predawn-to-noon sector where the spacecraft has a near-continuous view of the most intense midmorning SKR sources, in agreement with the Voyager findings, while elsewhere the SKR modulation phase depends strongly on spacecraft local time, being in approximate antiphase with the midmorning sources in the postdusk sector. Supporting evidence for this scenario is provided through an independent determination of the variable rotation period of the southern magnetic field perturbations throughout the 6 year interval.

Citation: Andrews, D. J., B. Cecconi, S. W. H. Cowley, M. K. Dougherty, L. Lamy, G. Provan, and P. Zarka (2011), Planetary period oscillations in Saturn's magnetosphere: Evidence in magnetic field phase data for rotational modulation of Saturn kilometric radiation emissions, J. Geophys. Res., 116, A09206, doi:10.1029/2011JA016636.

\section{Introduction}

[2] Powerful $\sim 10^{9} \mathrm{~W}$ broadband radio emissions from Saturn centered in the kilometer wave band, thus termed Saturn kilometric radiation (SKR), were first observed by the Planetary Radio Astronomy (PRA) experiments on the two Voyager spacecraft [Kaiser et al., 1980]. Circularly polarized waves were found to be emitted from the two hemispheres of the planet, predominately right handed from the north and left handed from the south, indicating emission in the extraordinary (X) mode [Warwick et al., 1981, 1982]. The power of the emissions was also found to be strongly modulated near the planetary rotation period, at a period of $\sim 10.7 \mathrm{~h}$ during

\footnotetext{
${ }^{1}$ Department of Physics and Astronomy, University of Leicester, Leicester, UK.

${ }^{2}$ LESIA, Observatoire de Paris, CNRS, UPMC, Université Paris VII Denis Diderot, Meudon, France.

${ }^{3}$ Blackett Laboratory, Imperial College, London, UK.

Copyright 2011 by the American Geophysical Union. 0148-0227/11/2011JA016636
}

the Voyager epoch [Desch and Kaiser, 1981], despite the lack of measureable azimuthal asymmetry in the internally generated planetary magnetic field [Smith et al., 1980; Connerney et al., 1982]. The phase of these modulations was also observed to be unchanged between the inbound and outbound passes of the Voyager flyby trajectories, at local times (LTs) near noon and dawn respectively, thus indicating a "strobe-like" (or "clock-like") modulation independent of the position of the observer, rather than a rotating source like a lighthouse beam [Warwick et al., 1981; Gurnett et al., 1981]. Similar strobe-like characteristics have also recently been found for lower frequency narrowband emissions in Saturn's magnetosphere, occurring in lagging quadrature with the SKR modulations [Wang et al., 2010; Ye et al., 2010].

[3] Due to the lack of directivity of the Voyager antenna system in the SKR band, centered typically near $\sim 200 \mathrm{kHz}$, the source locations could not be determined directly from the PRA data. However, these were inferred from various considerations of the beaming and visibility of the emissions along the spacecraft trajectories [Kaiser et al., 1981; Kaiser and Desch, 1982; Lecacheux and Genova, 1983]. Assuming fixed sources emitting near the $\mathrm{X}$ mode cutoff frequency (i.e., 
close to the local electron gyrofrequency) due to the cyclotron maser instability [Wu and Lee, 1979; Zarka, 1998], highlatitude auroral source locations were inferred in both hemispheres, extending typically from the midmorning sector toward noon [Galopeau et al., 1995].

[4] Subsequent studies have extended these initial results in two important respects. First, it has been shown, initially using Ulysses data and in more detail with Cassini, that the SKR modulation period varies slowly over time, typically by fractions of a percent over yearly intervals, much too large a rate to be associated directly with the rotation of the planet itself [Galopeau and Lecacheux, 2000; Gurnett et al., 2005; Kurth et al., 2007, 2008]. Moreover, it has also been demonstrated that the modulation periods corresponding to emissions from the northern and southern hemispheres are distinctly different, $\sim 10.6 \mathrm{~h}$ in the north and $\sim 10.8 \mathrm{~h}$ in the south during the southern summer conditions that prevailed in the initial preequinox Cassini era, 2004-2009 [Gurnett et al., 2009a, 2010b; Lamy, 2011]. Second, it has also been demonstrated from visibility considerations and use of the directionfinding capability of the Cassini Radio and Plasma Wave Science (RPWS) experiment [Gurnett et al., 2004], that SKR emission sources are not confined to the dawn-to-noon sector [Farrell et al., 2005; Cecconi et al., 2009]. Rather, they are broadly distributed around a circumpolar oval in each hemisphere that is coincident with the ultraviolet (UV) auroral oval [Lamy et al., 2009]. However, the emitted power strongly maximizes in the midmorning sector as found from Voyager results, where UV auroral emissions are also usually brightest [Trauger et al., 1998; Clarke et al., 2005; Grodent et al., 2005; Gérard et al., 2006]. Most recently it has been shown that the UV emission power in the dawn sector is also modulated near the planetary period, in phase with the SKR emissions from the corresponding hemisphere [Nichols et al., 2010a].

[5] The finding of circumpolar auroral SKR sources raises the issue of whether these emissions are modulated near the planetary period at all points around the oval, and if so, how the modulation phase may depend on LT. We note in this regard that while the brighter UV emissions in the dawn sector are modulated in phase with the overall SKR power in each hemisphere as just indicated, the weaker UV emissions in the dusk sector are instead modulated in antiphase with the overall SKR power [Nichols et al., 2010a]. This result suggests that the UV emissions, and possibly the SKR emissions as well, do not vary in concert around the oval, but rather in antiphase on opposite sides of the oval, at least between the stronger sources at dawn and the weaker sources at dusk. Initial direct evidence for rotating modulations of southern hemisphere SKR emissions has recently been obtained from direction-resolved Cassini data [Lamy, 2011].

[6] In addition to modulations in the emitted SKR and UV power, oscillations near the planetary period in plasma and field parameters have also been found to be ubiquitous throughout Saturn's magnetosphere, including the magnetic field [Espinosa and Dougherty, 2000; Espinosa et al., 2003a, 2003b; Cowley et al., 2006; Giampieri et al., 2006; Southwood and Kivelson, 2007; Andrews et al., 2008, 2010a, 2010b; Khurana et al., 2009; Provan et al., 2009a, 2011; Southwood, 2011], the magnetospheric cold and hot plasma populations and associated energetic neutral atom emissions [Carbary and Krimigis, 1982; Krupp et al., 2005; Gurnett et al., 2007, 2010a; Carbary et al., 2007a, 2007b, 2007c, 2008a, 2008b, 2009a, 2009b, 2010; Arridge et al., 2008; Burch et al., 2009], the location of the UV auroral oval [Nichols et al., 2008, 2010b; Provan et al., 2009b], the magnetopause and bow shock [Clarke et al., 2006, 2010a, 2010b], and auroral hiss emissions [Gurnett et al., 2009b]. In these cases, however, the perturbations rotate around the planet as an " $m=1$ " oscillation (thus varying with azimuthal angle $\varphi$ as $e^{-j \varphi}$ ), with a synodic period that is close to the SKR period. Magnetic field and auroral hiss modulations observed at high latitudes are found to have the same period as the SKR modulations in the corresponding hemisphere [Andrews et al., 2010b; Gurnett et al., 2009b], while the oscillations in the equatorial region contain both periods [Provan et al., 2011], but are dominated by the southern period during the preequinox intervals investigated in most of the above studies.

[7] In this paper we focus on the magnetic field oscillations near the planetary period observed in Saturn's magnetosphere, the form of which is sketched in Figures $1 \mathrm{a}$ and $1 \mathrm{~b}$, taken from Andrews et al. [2010b]. Here the black dashed lines indicate the near-axisymmetric "background" magnetospheric field with closed field lines at lower latitudes (gray) and open field lines in the polar regions (clear), while the red lines in Figure 1a and the green lines in Figure $1 \mathrm{~b}$ indicate the perturbation fields in the meridian planes shown associated with the southern and northern period systems, respectively. The perturbation field lines out of these planes can then be obtained to a first approximation simply by displacing the field loops shown directly into and out of the plane of the figure. The two patterns of perturbation fields then rotate independently around the central axis with a period close to the southern SKR period in Figure 1a, and close to the northern SKR period in Figure 1b. In the polar regions the perturbation fields are similar in form to that of a planetcentered rotating transverse dipole in each hemisphere [Provan et al., 2009a], the instantaneous effective directions of which are shown by the large planet-centered colored arrows in each figure. The near-equatorial field perturbations then take the form of rotating quasi-uniform fields lying approximately parallel to the equatorial plane in the directions of these effective dipoles, combined with north-south fields such that the perturbation field lines are arched with apices pointing to the north for the southern period perturbations, and pointing to the south for the northern period perturbations [Andrews et al., 2008, 2010a]. As indicated above, the southern and northern period perturbations are superposed in the equatorial magnetosphere, but with the southern oscillations being dominant by factors of $\sim 3$ during the preequinox Cassini interval [Provan et al., 2011]. The presence of the weaker northern period oscillations is detected via significant apparent "jitter" in the phase of the stronger southern period field oscillations.

[8] As discussed by Andrews et al. [2010b], the form of these perturbation fields, quasi-uniform in the equatorial region and quasi-dipolar at high latitudes, suggests the presence of two independent auroral current systems that rotate in the two hemispheres with differing periods. These are illustrated in Figures $1 \mathrm{c}$ and 1d, showing the current systems corresponding to Figures $1 \mathrm{a}$ and $1 \mathrm{~b}$ as viewed from 

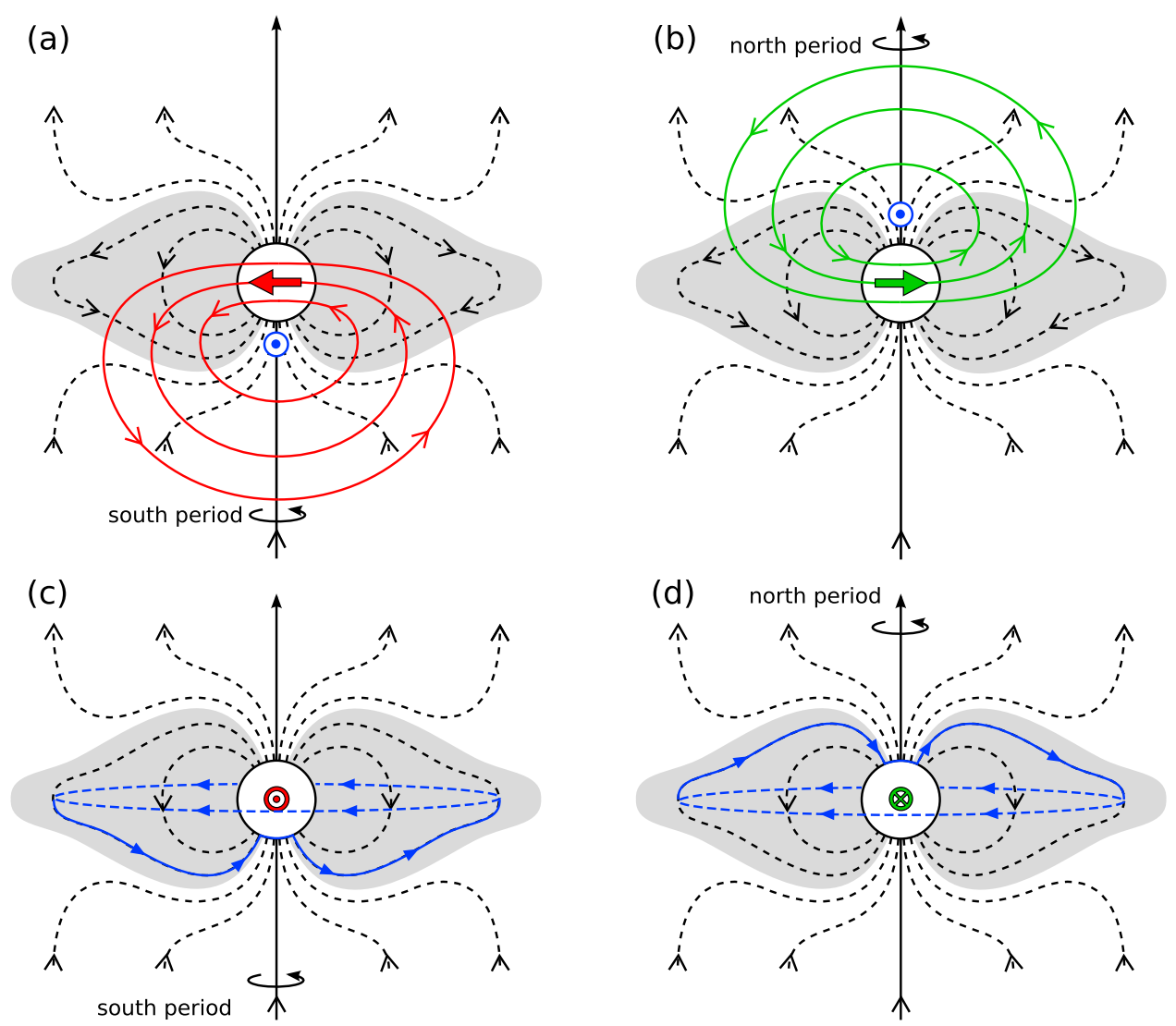

Figure 1. Sketches showing the form of the oscillatory perturbation fields deduced from the results of Andrews et al. [2010b], together with the implied current systems. (a and b) The principal meridian of the perturbation at a given instant (i.e., the meridian containing the perturbation field lines), where the black dashed lines show the quasi-static "background" magnetospheric field, with closed field lines at lower latitudes (gray region) and open field lines at higher latitudes (white region) mapping into the northern and southern polar regions. The perturbation field lines are then indicated by the colored solid lines, red for the southern system in Figure 1a and green for the northern system in Figure 1b. To a first approximation, the perturbation field lines out of the planes shown can be obtained simply by displacing the perturbation field loops directly into and out of the plane of the figure. The direction of the effective centered transverse dipole corresponding to the quasi-dipolar perturbation field in the open field region is shown by the solid arrow, while the sense of the current associated with the overall field perturbations given by Ampère's law is indicated by the blue symbols, directed out of the plane in both diagrams. The patterns shown correspond approximately to SKR maxima when the Sun is on the right in both figures, such that at these times the associated current flows approximately from dusk to dawn. A more detailed view of the current systems associated with Figures (c) $1 \mathrm{a}$ and (d) $1 \mathrm{~b}$ is given, where Figures $1 \mathrm{a}$ and $1 \mathrm{~b}$ are both viewed from the left. Field-aligned and ionospheric currents flowing from left to right in each figure are indicated by the solid blue lines, while the return currents flowing from right to left through the equatorial region outside the quasidipolar equatorial magnetosphere are indicated by the dashed blue lines. These perturbation field patterns and current systems then rotate independently in time approximately rigidly about the central axis at close to the southern SKR period in Figures 1a and 1c, and close to the northern SKR period in Figures $1 \mathrm{~b}$ and $1 \mathrm{~d}$, as indicated. (From Andrews et al. [2010b].)

the left. At any instant, currents flow into the auroral ionosphere in one LT sector, around and/or across the oval, and out of the opposite LT sector, closing in the equatorial magnetosphere. The relative phasing of the magnetic oscillations is such that the southern period quasi-uniform equatorial field and effective high-latitude transverse dipole both point down tail (and somewhat toward dawn) at southern SKR maxima, while the northern period quasi-uniform equatorial field and effective high-latitude transverse dipole both point sunward at northern SKR maxima [Andrews et al., 2010b; Provan et al., 2011]. These configurations correspond to those shown in Figures 1a and $1 \mathrm{~b}$ if the Sun is on the right in both cases, thus implying that SKR maxima in both hemispheres correspond to times at which the rotating currents flow out of the ionosphere in the dawn sector and into the ionosphere in the dusk sector [Andrews et al., 2010b]. Enhanced upward 
currents in the dawn sector then provide favorable conditions for enhanced downward-directed auroral electron acceleration and UV emission in that sector consistent with the results of Nichols et al. [2010a], together with enhanced SKR wave growth [Mutel et al., 2010]. The dominance of the modulated UV and SKR emissions from the dawn sector may then result from the combination of this rotating current system with quasi-steady upward currents in this sector resulting from the solar wind interaction [Southwood and Kivelson, 2009]. Simultaneously enhanced downward currents in the dusk sector may then reduce auroral acceleration and UV emission in that sector, also consistent with Nichols et al. [2010a], together potentially with corresponding SKR emission. On the other hand, enhanced downward currents will tend to enhance auroral hiss emissions in the dusk sector during such intervals, produced by upward moving electron beams, consistent with the results of Gurnett et al. [2009b]. The latter authors show that the rotating auroral hiss modulations maximize in the dusk sector in each hemisphere at the times of corresponding SKR maxima.

[9] Overall, therefore, the existence of rotating magnetic perturbations implying the existence of rotating current systems in the two hemispheres also suggests the occurrence of rotating modulations in the SKR emissions, corresponding to (but in approximate antiphase with) the rotating modulations in auroral hiss. If an observer could then in effect see all of the SKR emission sources at any instant, the combined modulations would correspond to those of the most intense sources in the midmorning sector, giving a strobelike modulation overall whose phase is independent of the position of the observer, as reported by Warwick et al. [1981] and Gurnett et al. [1981]. However, in practice the visibility of the sources around the oval is strongly constrained by the beaming of the radiation, which is emitted from the "source surface" near the corresponding electron gyrofrequency into an outward facing hollow cone of angles around the field direction [Cecconi et al., 2009; Lamy et al., 2008b, 2009, 2010]. With a cone angle relative to the field of typically $\sim 70^{\circ}$ and an angular width of only $\sim 5^{\circ}$ about that direction, modeling and observations show that at typical near-equatorial spacecraft locations emissions near the peak in the SKR spectrum can only be observed from two narrow bands $\sim 1 \mathrm{~h}$ LT wide around the oval, displaced $\sim 3-4 \mathrm{~h}$ LT on either side of the spacecraft location [Lamy et al., 2008b, 2009; Cecconi et al., 2009]. This implies that the spacecraft can view the most intense SKR sources in the midmorning sector, in one or other of these two azimuthal "windows," only over a relatively restricted range of LTs extending roughly $4 \mathrm{~h}$ to either side of these sources, thus typically spanning the predawn to postnoon sector. Within this range of LTs the observed modulations will thus be dominated by the midmorning sources, giving rise to strobe-like modulations approximately independent of LT. This conclusion is then consistent with the Voyager findings, whose inbound and outbound passes were located near noon and dawn, respectively. Away from the predawn to postnoon sector, however, the observed emissions will arise from other LT sectors around the oval, potentially giving rise to positiondependent SKR modulation phases if these modulations indeed rotate around the oval like the field and plasma perturbations. We note that Zarka et al. [2007] previously pro- vided evidence that the SKR period varies somewhat with the orbital motion of the spacecraft.

[10] This discussion may then be relevant to a significant unresolved issue concerning the existence of slow long-term phase drifts between the southern period magnetic field oscillations at both high and low latitudes and the related southern SKR modulations. These phase drifts are typically only a few tenths of a degree per day, both positive and negative in different intervals, corresponding to apparent differences in period of a few tens of seconds. However, they integrate over intervals of hundreds of days to relative phase shifts of several tens of degrees. Such variations have been evident from the initial investigations of the relative phases between the SKR and magnetic field oscillations [Andrews et al., 2008; Provan et al., 2009a], and remain present at comparable values using the most recent phase determinations [Andrews et al., 2010b]. We note, however, that phase drift effects of comparable magnitude do not appear in the northern high-latitude field oscillations relative to the northern SKR modulations, though the northern oscillations have been significantly less continuously monitored by Cassini than have the southern period oscillations. A possible explanation for the relative phase drift in the southern system lies in the anticipated slow variations of SKR modulation phase due to the position-dependent effects outlined above, as the orbit of the spacecraft around the planet evolves during the mission. In this paper we develop a simple theoretical model of the SKR phase shifts that might be expected on this basis, and examine whether these could give rise to the relative phase drifts observed.

\section{Methodology}

\subsection{Theoretical Model of Position-Dependent SKR Modulation Phases}

[11] In Figure 2a we show the variation of the SKR intensity with azimuth $\varphi$ around Saturn's auroral oval, normalized to the peak intensity, derived from the direction-finding study of Lamy et al. [2009]. Northern and southern emissions were found to yield similar results, and are shown combined in this plot. Here azimuth $\varphi$, equivalent to LT, is measured from noon positive toward dusk as in previous related studies [Andrews et al., 2008, 2010a, 2010b; Provan et al., 2009a, 2011], such that dusk corresponds to $90^{\circ}$ and dawn to $270^{\circ}$, as marked at the top. The peak in the emission intensity in the midmorning sector is evident. We then consider the emitted SKR power per unit azimuthal angle, $p(\varphi)$, which we take to be proportional to the quantity shown, and assume for simplicity that the periodic modulations in power around the oval correspond to some fixed fraction $k$ of this power. Following the discussion in section 1 related to rotating field and current systems, we also assume that the power modulations rotate as an $m=1$ oscillation around each auroral oval, such that we can write for the modulations $\delta p$

$$
\delta p(\varphi, t)=k p(\varphi) \mathrm{e}^{j\left(\Phi_{S K R}(t)-\varphi\right)},
$$

where $j=\sqrt{-1}$ and we take the real part of the complex function. The phase function $\Phi_{S K R}(t)$ defines the slowly variable SKR modulation period (separately for each hemisphere, 
$\Phi_{S K R n}(t)$ and $\Phi_{S K R s}(t)$ for the north and south, respectively) through

$$
\tau_{S K R}(t)=\frac{360}{\left(\frac{d \Phi_{S K R}}{d t}\right)}
$$

where the phase function is expressed in degrees, and its absolute value is such that SKR modulation maxima occur at noon $\left(\varphi=0^{\circ}\right)$ when

$$
\Phi_{S K R}(t)=360 N \mathrm{deg},
$$

where $N$ is any integer.
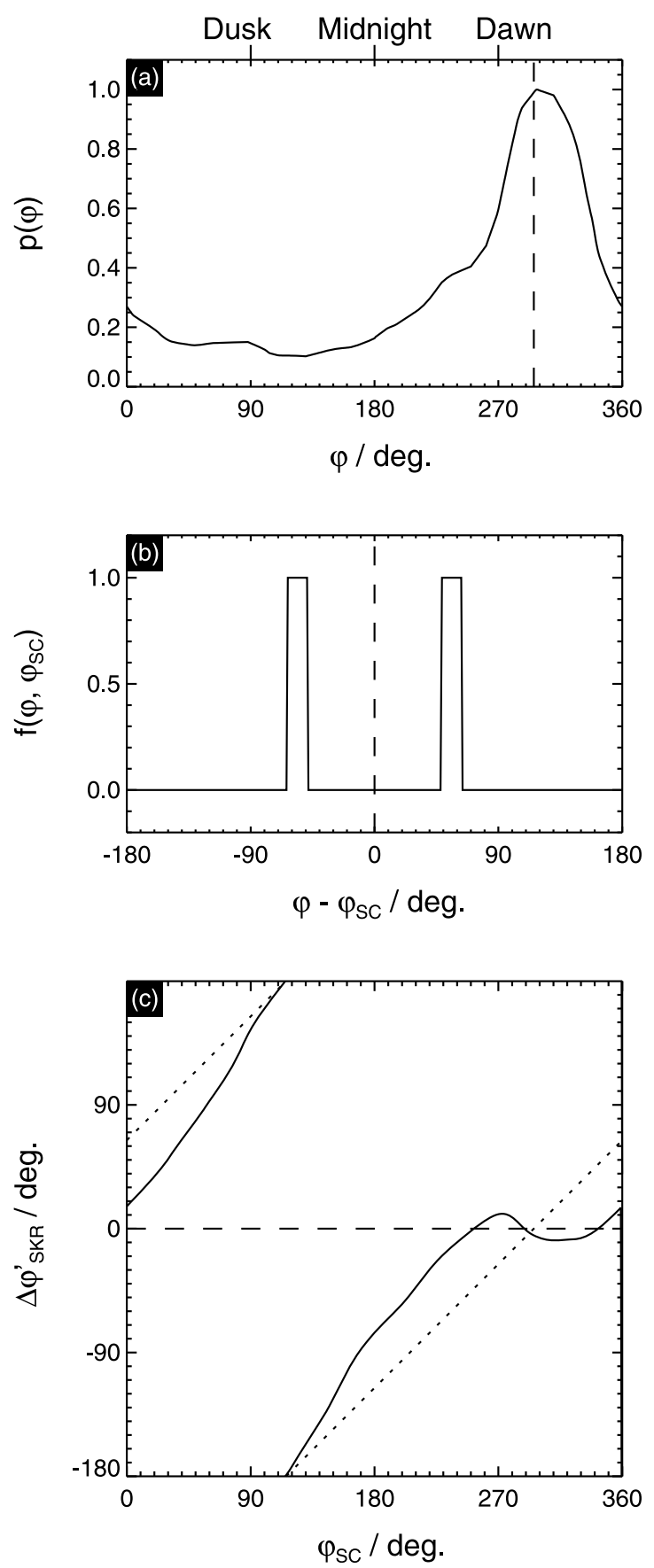

[12] If an observer can then see all of the sources present, independent of azimuth, the power modulations integrated over all the sources will be

$$
\begin{aligned}
\delta P(t) & =\int_{0^{\circ}}^{360^{\circ}} \delta p(\varphi, t) d \varphi=k e^{j \Phi_{S K R}(t)} \int_{0^{\circ}}^{360^{\circ}} p(\varphi) e^{-j \varphi} d \varphi \\
& =k P_{S K R} \mathrm{e}^{j\left(\Phi_{S K R}(t)-\varphi_{S K R}\right)},
\end{aligned}
$$

where we have written

$$
\int_{0^{\circ}}^{360^{\circ}} p(\varphi) e^{-j \varphi} d \varphi=P_{S K R} \mathrm{e}^{-j \varphi_{S K R}},
$$

where $P_{S K R}$ is pure real, the modulus of the integral, and argument phase angle $\varphi_{S K R}$ is the effective azimuth corresponding to the total integrated SKR modulation. Application of equation ( $2 b)$ to the azimuthal power profile shown in Figure $2 \mathrm{a}$ yields the value $\varphi_{S K R} \simeq 296^{\circ}$ corresponding to a LT of $\sim 7.7 \mathrm{~h}$ in the postdawn sector, marked by the vertical dashed line in the figure. As expected, this azimuth corresponds closely to that of the peak in the SKR emission intensity. The "true strobe" (or "clock") phase of the SKR modulation may thus be taken to be

$$
\Phi_{S K R}^{*}(t)=\Phi_{S K R}(t)-\varphi_{S K R},
$$

such that the total integrated power exhibits maxima when

$$
\Phi_{S K R}^{*}(t)=360 N \operatorname{deg},
$$

where $N$ is any integer.

[13] Following the discussion in section 1, however, we now consider that due to the conical beaming of the radio emissions, a given observer has only a restricted view of the sources distributed around the auroral oval. Specifically, on the basis of the results presented by Lamy et al. [2008b, 2009] and Cecconi et al. [2009] we suppose that emission

Figure 2. (a) A profile of the SKR emitted power $p(\varphi)$ around Saturn's auroral oval versus azimuth $\varphi$, derived from northern and southern emissions combined by Lamy et al. [2009, Figure 5]. The values shown here have been normalized to the peak value. The vertical dashed line indicates the effective azimuth of the combined "true strobe" emissions, $\varphi_{S K R} \simeq 296^{\circ}$, determined from equation (2b). (b) The model SKR "seeing function" $f\left(\varphi, \varphi_{s c}\right)$ around the auroral oval employed in this paper, corresponding to two windows $15^{\circ}$ ( $1 \mathrm{~h} \mathrm{LT})$ of azimuth wide, centered $\pm 55^{\circ}(\sim 3.7 \mathrm{~h} \mathrm{LT})$ on either side of the spacecraft azimuth $\varphi_{s c}$. Function $f$ is shown plotted versus $\varphi-\varphi_{s c}$. (c) The SKR modulation phase deviation $\Delta \varphi_{S K R}^{\prime}$ due to restricted "seeing" of the SKR sources around the auroral oval defined by equations (3a)-(3e) (solid line), plotted versus spacecraft azimuth $\varphi_{s c}$. The horizontal dashed line shows the true strobe limit $\Delta \varphi_{S K R}^{\prime}=0$ corresponding to full visibility of all SKR sources, while the inclined dotted line shows the opposite limit $\Delta \varphi_{S K R}^{\prime}=\varphi_{S c}-\varphi_{S K R}$ in which emissions are detected only from subspacecraft sources such that the modulation phase corresponds to the LT of the spacecraft. 
sources near the peak of the SKR spectrum (few hundred $\mathrm{kHz}$ ) that contribute most to the observed power modulation are confined to two narrow bands of LT $\sim 1 \mathrm{~h}$ wide, displaced $\sim 4 \mathrm{~h}$ LT on either side of the spacecraft position. This picture applies to the usual near-equatorial Cassini observing positions extending well outside the $\sim 4 \mathrm{R}_{\mathrm{S}} \mathrm{SKR}$ shadow zone [Lamy et al., 2008a, 2008b], to typical radial distances of a few tens of $R_{S}$. $\left(R_{S}\right.$ is Saturn's radius equal to $60268 \mathrm{~km}$.) We thus define a "seeing function" for the SKR emissions around the oval that depends principally on the azimuth $\varphi_{s c}$ of the spacecraft, $f\left(\varphi, \varphi_{s c}\right)$, whose value lies between 0 and 1 , and which for simplicity we take to consist of two top hats where $f=1$, each of azimuthal width $15^{\circ}(1 \mathrm{~h} \mathrm{LT})$, centered at $\varphi=\varphi_{s c} \pm 55^{\circ}\left(\varphi_{s c} \pm 3.7 \mathrm{~h} \mathrm{LT}\right)$, with $f=0$ otherwise. This form of $f$ is shown in Figure 2b, plotted versus $\varphi-\varphi_{s c}$.

[14] The observed SKR power modulations are then given by

$$
\begin{aligned}
\delta P^{\prime}\left(\varphi_{s c}, t\right) & =\int_{0^{\circ}}^{360^{\circ}} f\left(\varphi, \varphi_{s c}\right) \delta p(\varphi, t) d \varphi \\
& =k e^{j \Phi_{S K R}(t)} \int_{0^{\circ}}^{360^{\circ}} f\left(\varphi, \varphi_{s c}\right) p(\varphi) e^{-j \varphi} d \varphi \\
& =k P_{S K R}^{\prime} e^{j\left(\Phi_{S K R}(t)-\varphi_{S K R}^{\prime}\left(\varphi_{s c}\right)\right)}
\end{aligned}
$$

where we have now written

$$
\int_{0^{\circ}}^{360^{\circ}} f\left(\varphi, \varphi_{s c}\right) p(\varphi) e^{-j \varphi} d \varphi=k P_{S K R}^{\prime} \mathrm{e}^{-j \varphi_{S K R}^{\prime}\left(\varphi_{s c}\right)}
$$

equivalent to equation ( $2 b)$ for the fully visible oval when $f=1$. We note that primed symbols are employed here, and throughout the paper, to indicate "observed" quantities which depend on the seeing function $f$, and thus on the azimuth of the spacecraft. The modified position-dependent effective azimuth of the SKR modulations is thus $\varphi_{S K R}^{\prime}\left(\varphi_{s c}\right)$, such that the observed SKR modulation phase is now

$$
\Phi_{S K R}^{\prime}(t)=\Phi_{S K R}(t)-\varphi_{S K R}^{\prime}\left(\varphi_{s c}\right)
$$

where $\Phi_{S K R}^{\prime}(t)=360 N$ deg at observed SKR power maxima. The observed phase $\Phi_{S K R}^{\prime}(t)$ is thus related to the true strobe phase $\Phi_{S K R}^{*}(t)$ by

$$
\begin{aligned}
\Phi_{S K R}^{\prime}(t) & =\Phi_{S K R}^{*}(t)-\left(\varphi_{S K R}^{\prime}\left(\varphi_{s c}\right)-\varphi_{S K R}\right) \\
& =\Phi_{S K R}^{*}(t)-\Delta \varphi_{S K R}^{\prime}\left(\varphi_{s c}\right)
\end{aligned}
$$

such that the observed phase is shifted to smaller values relative to the true strobe phase through phase angle

$$
\Delta \varphi_{S K R}^{\prime}\left(\varphi_{s c}\right)=\varphi_{S K R}^{\prime}\left(\varphi_{s c}\right)-\varphi_{S K R}
$$

where $\varphi_{S K R}^{\prime}\left(\varphi_{S c}\right)$ and $\varphi_{S K R}$ are given by equations (3b) and (2b), respectively. In general we would expect the phase deviation $\Delta \varphi_{S K R}^{\prime}\left(\varphi_{s c}\right)$ to take positive values for observation points having later LTs than those of the most intense mid- morning sources centered at $\sim 7.7 \mathrm{~h} \mathrm{LT}$, such that observed maxima would then be shifted to somewhat later times, and to take negative values for observation points with earlier LTs than those of the most intense sources, such that observed maxima would then be shifted toward somewhat earlier times.

[15] Application of equations (3a)-(3e) with the two top hat seeing function to the power profile shown in Figure $2 \mathrm{a}$ yields the results shown in Figure 2c, where we show $\Delta \varphi_{S K R}^{\prime}$ plotted versus spacecraft azimuth $\varphi_{s c}$. The true strobe limit $\Delta \varphi_{S K R}^{\prime}=0$ corresponding to a complete view of all the SKR sources is indicated by the horizontal dashed line, while the inclined dotted line shows the opposite limit $\Delta \varphi_{S K R}^{\prime}=\varphi_{S c}-\varphi_{S K R}$ in which the modulation phase corresponds simply to that of the subspacecraft sources. In this case $\Delta \varphi_{S K R}^{\prime}$ passes through zero when the spacecraft azimuth passes through the effective azimuth of the total SKR emission $\varphi_{S K R} \simeq 296^{\circ}$, and reaches $\pm 180^{\circ}$ at the antipodal azimuth of $\sim 116^{\circ}$ in the postdusk sector $(\sim 19.7 \mathrm{~h} \mathrm{LT})$. The variation of $\Delta \varphi_{S K R}^{\prime}\left(\varphi_{S c}\right)$ computed using the seeing function in Figure $2 \mathrm{~b}$ is then shown by the solid line in Figure $2 \mathrm{c}$. It can be seen that this is indeed strobe like, remaining within $\pm 15^{\circ}$ of zero, over a $\sim 120^{\circ}$ range of LTs extending from the predawn sector to noon as anticipated in section 1 , thus being in agreement with the Voyager findings. Away from this sector, however, the modulation phase varies strongly with spacecraft LT, reaching antiphase with the most intense midmorning sources when the spacecraft is located at antipodal azimuths in the postdusk sector. The phase difference exceeds $\pm 90^{\circ}$ in a band of azimuths $\sim 100^{\circ}$ wide centered in this sector.

\subsection{Orbit-Averaged Phase Deviations}

[16] The results in Figure 2 imply in the first instance that significant deviations in SKR modulation phase will occur around the Cassini orbit on time scales of a few tens of days, though observations on a given orbit will generally be dominated by those near apoapsis where the spacecraft spends most time. However, as discussed in section 3.1 below, the SKR modulation periods (and hence phases) employed here and in previous studies were determined over significantly longer sliding intervals of 200 days, thus generally encompassing a significant number of individual orbits. As an initial step toward simulating the effect of the position-dependent phase deviations on such long-term values, here we first derive orbit-averaged phase deviations from Saturn Orbit Insertion (SOI) in July 2004 to Rev 134 in July 2010, the latter corresponding to approximately one year after Saturn equinox.

[17] We begin by outlining the evolution of the Cassini orbit over this interval. In Figures $3 a$ and $3 b$ we show a representative selection of seven spacecraft orbits (Revs) plotted from apoapsis to apoapsis, projected into the $X-Y$ and $Y-Z$ planes, respectively. The coordinate system is such that $Z$ is aligned with the planet's spin and magnetic axes, the $X-Z$ plane contains the Sun, and $Y$ points toward dusk, completing the right-handed set. The Sun is therefore at the top of the plots in Figure $3 \mathrm{a}$ and into of the plane of the plots in Figure $3 \mathrm{~b}$, while dusk and dawn are to the left and right, respectively, in both. Each plot box has sides of length $90 R_{S}$, but the position of the planet (the origin) has been adjusted as required. Day of year is indicated around each orbit at 3 day intervals where space allows, with the year concerned and 


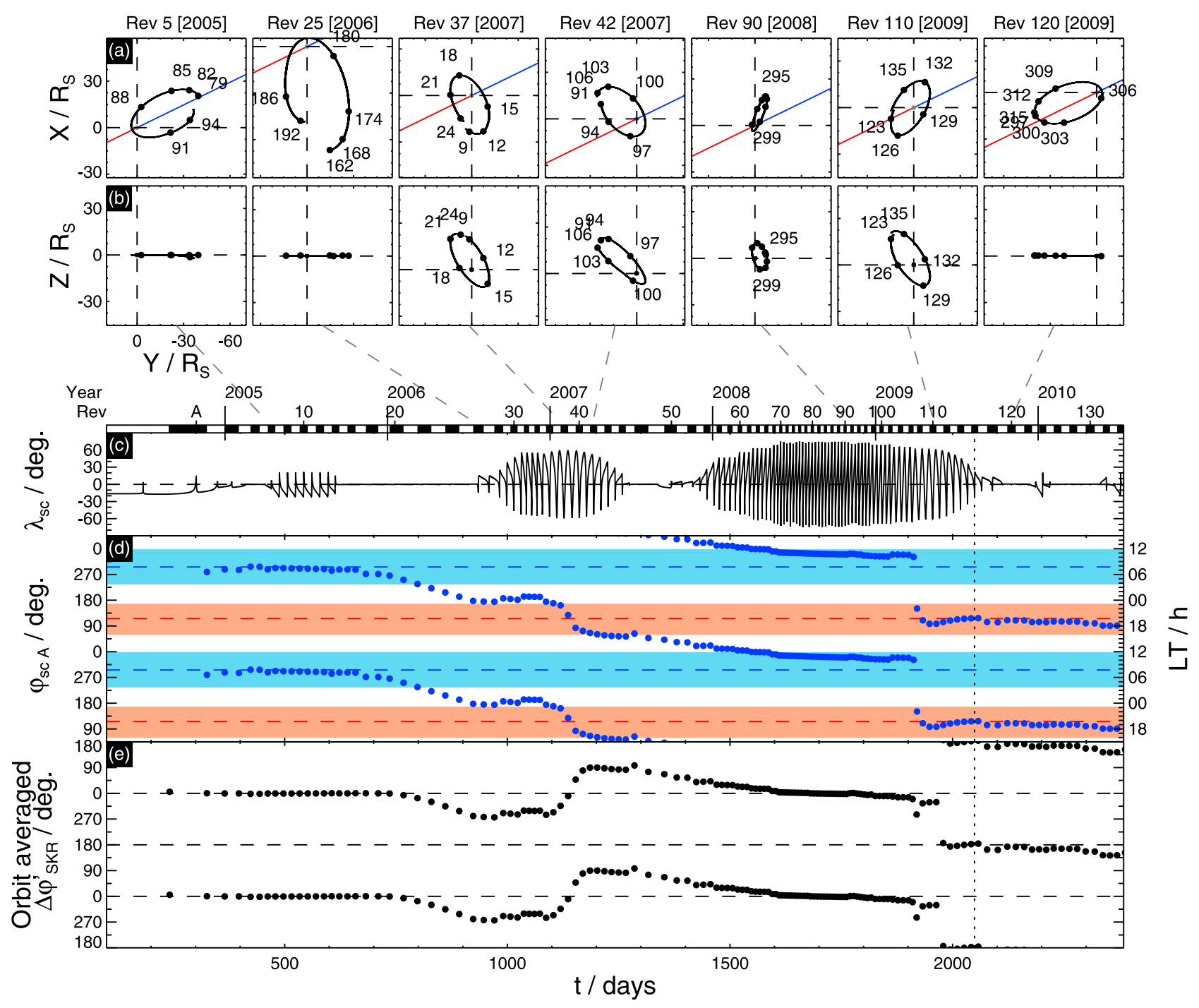

Figure 3. Projections of the spacecraft trajectory onto the (a) $X-Y$ (equatorial) and (b) $Y-Z$ (dawn-dusk meridian) planes for a selection of seven Cassini Revs. The orthogonal dashed lines show the corresponding coordinate axes passing through the planet's center, whose position is varied between plots to best display the orbit, with the plot scale $\left(90 \mathrm{R}_{\mathrm{S}}\right.$ on each side) held fixed. Day of year numbers are indicated by the solid circles along the trajectory at 3 day intervals where space allows, while calendar year and Rev numbers are given at the top of each pair of plots. The blue and red lines in Figure 3 a indicate the effective azimuth of the true strobe SKR source, and its antipodal azimuth, respectively. (c-d) Time series plots over the interval $t=100-2384$ days (SOI to Rev 134), where $t=0$ days corresponds to 00 UT on 1 January 2004. Saturn vernal equinox at $t=2049$ days is indicated by the vertical dotted line. The duration of each Rev from apoapsis to apoapsis is indicated at the top by the row of alternate black and white blocks, with every tenth Rev numbered. Calendar year boundaries are also marked. Figure $3 \mathrm{c}$ shows the latitude of the spacecraft $\lambda_{s c}$ (degrees). Figure 3d shows the azimuth of spacecraft apoapsis $\varphi_{s c}$ (degrees), with two cycles being shown to emphasize the continuity of the data (blue dots), such that each point is plotted twice. Equivalent LT (h) is indicated on the right-hand axis. The blue and red dashed lines indicate the effective azimuth of the true strobe SKR source $\varphi_{S K R}$ and its antipodal azimuth, respectively. The blue shaded region indicates the azimuthal range about the blue dashed line where the SKR modulation phase differs from the true strobe phase by less than $\pm 15^{\circ}$, i.e., where $\left|\Delta \varphi_{S K R}^{\prime}\right| \leq 15 \mathrm{deg}$, while the red shaded region indicates the azimuthal range about the red dashed line where the SKR phase deviation exceeds $\pm 90^{\circ}$, i.e., where $\left|\Delta \varphi_{S K R}^{\prime}\right| \geq 90$ deg. Figure 3e shows the directional mean of the modeled SKR phase deviation values $\Delta \varphi_{S K R}^{\prime}$, computed over each orbit from periapsis to periapsis, with two complete cycles of phase again being shown. 
Rev number (defined from apoapsis to apoapsis) being given at the top of each plot. The blue line in the equatorial plots in Figure $3 \mathrm{a}$ shows the effective azimuth of the total SKR emission $\varphi_{S K R} \simeq 296^{\circ}$, while the antipodal azimuth $\sim 116^{\circ}$ is indicated by the red line.

[18] Figures $3 \mathrm{c}$ and $3 \mathrm{~d}$ then show orbit parameters versus time $t$ (days) spanning SOI through Rev 134. As for all the time series plots in this paper, the plot covers the interval from $t=100$ days (10 April 2004) to 2384 days (12 July 2010), where $t=0$ days corresponds to 00 UT on 1 January 2004. The time of Saturn's vernal equinox at $t=2049$ days (11 August 2009) is indicated by the vertical black dotted line. Calendar year boundaries are indicated along the top of Figure 3c, along with alternate black and white blocks indicating the duration of each Rev, numbered every ten Revs. Gray dashed lines connect each pair of orbit plots in Figures $3 \mathrm{a}$ and $3 \mathrm{~b}$ to their corresponding Rev at the top of Figure $3 \mathrm{c}$. In the Figure $3 \mathrm{c}$ we plot the latitude of the spacecraft $\lambda_{s c}$ versus time, showing the presence of two extended intervals of high-latitude orbits, with the orbit otherwise remaining close to equatorial. The first high-latitude interval spans approximately from $\operatorname{Rev} 30$ to $\operatorname{Rev} 43(t \approx 1000-1200$ days), and the second from $\operatorname{Rev} 59$ to $\operatorname{Rev} 113(t \approx 1500-2000$ days $)$. Measurements of the polar perturbation magnetic fields are confined to within these intervals.

[19] Figure $3 \mathrm{~d}$ shows the azimuth of apoapsis (blue circles) of each orbit, $\varphi_{s c A}$, where for purposes of continuity two complete $360^{\circ}$ cycles are shown. Equivalently, the LT of apoapsis is indicated on the right-hand axis. For comparison, the horizontal dashed lines show the effective azimuth corresponding to the true strobe $\mathrm{SKR}$ emission $\varphi_{S K R} \simeq 296^{\circ}$ (blue) and its antipodal azimuth $\sim 116^{\circ}$ (red), corresponding to the red and blue lines in the equatorial plots in Figure 3a. The blue shaded area then shows the azimuthal extent about the blue dashed line where the SKR modulation is expected to be closely strobe like, with the modulation phase lying within $\pm 15^{\circ}$ of the true strobe phase (i.e., $\left|\Delta \varphi_{S K R}^{\prime}\right| \leq 15^{\circ}$ ), while the red shaded area shows the extent about the red dashed line where the SKR modulation deviates substantially from strobe-like behavior, with the modulation phase lying beyond $\pm 90^{\circ}$ of the true strobe phase (i.e., $\left|\Delta \varphi_{S K R}^{\prime}\right| \leq 90^{\circ}$ ). It can be seen that for the first $\sim 5$ years of the mission, up to $t \approx 1900$ days corresponding to the first quarter of 2009 , the apoapsis of the orbit, where the spacecraft spends most time, was almost continuously located outside the red shaded region, except for a brief interval around $t \approx 1150$ days (Rev 38) when apoapsis passed rapidly through the antipodal azimuth. The SKR modulation phase should thus remain close to that of the most intense midmorning sources throughout this period, except possibly during the latter short interval. However, a significant change of orbit geometry took place after $t \approx 1900$ days, when apoapsis was in effect exchanged with periapsis via a near-circular orbit (Rev 108). This maneuver resulted in a rapid $\sim 180^{\circ}$ switch in the azimuth of apoapsis to values nearly antipodal to $\varphi_{S K R}$ lying centrally within the red shaded band, a configuration maintained to the end of the interval studied here. The results in Figure 2 then suggest the possibility of large deviations of SKR modulation phase from the true strobe phase during this later interval, when the spacecraft was principally observing SKR sources on the opposite side of the planet to the most intense midmorning sources.
[20] The resulting orbit-averaged values of $\Delta \varphi_{S K R}^{\prime}$ are shown versus time in Figure $3 \mathrm{e}$, where we again show two complete $360^{\circ}$ cycles of phase for purposes of continuity. These values have been obtained by suitably averaging the phase deviations $\Delta \varphi_{S K R}^{\prime}\left(\varphi_{s c}\right)$ computed from equations (3a) (3e) at $1 \mathrm{~min}$ resolution for each orbit from periapsis to periapsis, with the resulting value being plotted at the mean time of each orbit near the time of corresponding apoapsis. We note that no account has been taken in this procedure of possible spacecraft entry into the "shadow zone" where SKR emissions cannot be observed [Lamy et al., 2008a, 2008b], which corresponds only to very short intervals near periapsis on orbits whose periapsis radius is sufficiently small (less than $\sim 4 \mathrm{R}_{\mathrm{S}}$ in the equatorial region). With regard to the "averaging" concerned, as for any cyclic quantity, the standard "arithmetic mean" is not appropriate to these phase angle data. Rather, we employ the "directional mean" [e.g., Mardia and Jupp, 2000], in which we separately average the sine and cosine of the phase values, and then compute the two-argument arctangent of the result. Precisely, if the phase deviation values to be averaged are $\Delta \varphi_{S K R k}^{\prime}$ for $k=1$ to $K$, the directional mean $\overline{\Delta \varphi_{S K R}^{\prime}}$ is given by the argument of the sum

$$
\frac{1}{K} \sum_{k=1}^{K} e^{j \Delta \varphi_{S K R}^{\prime} k}=\overline{R_{\Delta \varphi_{S K R}^{\prime}}} e^{\overline{\Delta \varphi_{S K R}^{\prime}}}
$$

where modulus $\overline{R_{\Delta \varphi_{S K R}^{\prime}}}$ (lying between values of 0 and 1 ), termed the "mean resultant length," gives a measure of the dispersion of the values about the mean. A brief overview of some principal features of "directional statistics" germane to this paper is provided in Appendix A.

[21] It is readily seen from Figure $3 e$ that near-zero values of orbit-averaged $\Delta \varphi_{S K R}^{\prime}$ are obtained when the azimuth of apoapsis in Figure $3 \mathrm{~d}$ lies close to the blue shaded region centered on $\varphi_{S K R} \simeq 296^{\circ}$, implying that the overall modulation phase of the detected SKR emission is close to that of the most intense midmorning sources. However, larger phase deviations of up to $\sim \pm 0^{\circ}$ are obtained on either side of Rev 38 at $t \approx 1150$ days, reversing from negative to positive as the spacecraft passes through the red shaded region. Shortly after Rev 108 at $t \approx 1900$ days, however, as apoapsis moves into the postdusk sector, the orbit-averaged model phase then changes by $\sim 180^{\circ}$, corresponding to observations of SKR sources in the dusk-side oval $\sim 4 \mathrm{~h} \mathrm{LT}$ on either side of spacecraft apoapsis, which combine to produce an overall modulation which is in approximate antiphase with the most intense midmorning sources. Under these circumstances, the orientation of the oscillatory perturbation magnetic field is expected to be essentially reversed in sense at observed SKR maxima compared with that observed during the first $\sim 5$ years of the mission as reported, e.g., by Andrews et al. [2008, 2010a, 2010b] and Provan et al. [2009a], an effect that should be very evident in the magnetic field phase data.

\subsection{Effect of SKR Modulation Phase Deviations on Derived Magnetic Oscillation Phases}

[22] We thus directly consider the effect of the positiondependent SKR modulation phase deviations on the relative phases of the magnetic field oscillations. The latter are 
determined empirically by fitting filtered residual (internal planetary field removed) magnetic field data with the $m=1$ function

$$
B_{i}(\varphi, t)=B_{i 0} \cos \left(\Phi_{S K R}^{\prime}(t)-\varphi-\psi_{i}^{\prime}\right),
$$

such that we obtain the phase value $\psi_{i}^{\prime}$ for field component $i$ (to modulo $360^{\circ}$ ) for a given interval of data relative to the observed SKR phase $\Phi_{S K R}^{\prime}(t)$, used as an exact guide phase. As in previous analyses, we employ spherical polar field components in the radial $(r)$, colatitudinal $(\theta)$, and azimuthal $(\varphi)$ directions, referenced to Saturn's spin and magnetic axis. As described in section 3.2, the fits are made over spacecraft passes through particular magnetospheric regions (e.g., the inner quasi-dipolar equatorial region or on high-latitude open field lines in a given hemisphere) that usually last one or two days, thus encompassing two or more field oscillation cycles whose phase can reliably be determined to within a few degrees. It is these phase values $\psi_{i}^{\prime}$ that show the slow pass-to-pass phase drifts with time that were discussed in section 1 .

[23] The values of the phases $\psi_{i}^{\prime}$ define the orientation of the perturbation field throughout the SKR modulation cycle observed during a particular epoch. Specifically, from equation (5a) at observed SKR maxima (when $\Phi_{S K R}^{\prime}(t)=360 N$ deg), the oscillation in field component $i$ has a maximum at azimuth $\varphi_{B_{i}}^{\prime} \max$ given to modulo $360^{\circ}$ by

$$
\varphi_{B_{i} \max }^{\prime}=-\psi_{i}^{\prime}
$$

with the pattern then rotating through $360^{\circ}$ in the sense of planetary rotation during the SKR modulation cycle (given that the periods are almost equal). Consideration of Figure 1 then shows that for the southern system the upward fieldaligned current from the ionosphere is expected to be centered approximately $90^{\circ}$ ahead in azimuth of the maximum in the radial field component (at both low and high latitudes), with the downward current centered approximately $90^{\circ}$ in azimuth behind. For the northern system these directions are reversed. Thus at observed SKR maxima the rotating upward field-aligned current region is expected to be located in a broad azimuthal band centered near azimuth $\varphi_{u F A C}^{\prime}$ max given by

$$
\varphi_{u F A C \max }^{\prime} \approx-\psi_{r}^{\prime} \pm 90^{\circ}
$$

where the upper sign corresponds to the southern system and the lower to the northern. The phase relations with the other field components should then be consistent with the polarizations shown in Figure 1. If modulation of the SKR emission is positively correlated with modulation of the upward field-aligned current as expected, then equation (5c) indicates the central azimuth (or LT) of the SKR sources related to the observed modulation, potentially depending on position according to the earlier discussion in this section.

[24] If the SKR modulations are indeed linked to the same rotating current system that is responsible for the rotating magnetic field perturbations, however, we may expect that the magnetic field phase relative to the true strobe phase should be essentially constant in time. Substituting from equation (3d) into equation ( $5 \mathrm{a}$ ) to express the magnetic oscillations in terms of the true strobe phase $\Phi_{S K R}^{*}(t)$ then yields

$$
\begin{aligned}
B_{i}(\varphi, t) & =B_{i 0} \cos \left(\Phi_{S K R}^{*}(t)-\varphi-\left(\psi_{i}^{*}+\left\langle\Delta \varphi_{S K R}^{\prime}\left(\varphi_{s c}\right)\right\rangle\right)\right) \\
& =B_{i 0} \cos \left(\Phi_{S K R}^{*}(t)-\varphi-\psi_{i}^{*}\right)
\end{aligned}
$$

where $\left\langle\Delta \varphi_{S K R}^{\prime}(\varphi)\right\rangle$ is some suitably averaged value of the position-dependent SKR phase deviation. Details of the averaging employed here, related to the derivation of the SKR modulation phase from sliding 200 day intervals of data, is discussed in section 3.3. Magnetic phase $\psi_{i}^{\prime}$ relative to the observed SKR modulation phase $\Phi_{S K R}^{\prime}(t)$ and phase $\psi_{i}^{*}$ relative to the true strobe phase $\Phi_{S K R}^{*}(t)$ are thus related by

$$
\psi_{i}^{\prime}=\psi_{i}^{*}-\left\langle\Delta \varphi_{S K R}^{\prime}\left(\varphi_{s c}\right)\right\rangle
$$

where $\psi_{i}^{*}$ is expected to be a constant. As the spacecraft orbit then evolves slowly with time, such that the value of $\left\langle\Delta \varphi_{S K R}^{\prime}\left(\varphi_{s c}\right)\right\rangle$ also evolves slowly with time, then so too will the phase $\psi_{i}^{\prime}$ of the magnetic field oscillations relative to the observed SKR modulations, leading to the appearance of long-term relative phase drifts in the opposite sense to $\left\langle\Delta \varphi_{S K R}^{\prime}\left(\varphi_{s c}\right)\right\rangle$. The values of $\psi_{i}^{*}$ for field component $i$, obtained from equation (5e) through modeling the averaged SKR phase deviation $\left\langle\Delta \varphi_{S K R}^{\prime}\left(\varphi_{s c}\right)\right\rangle$, then define the orientation of the perturbation field throughout the true strobe SKR modulation cycle, constant over time. Specifically, in correspondence with equation ( $5 b)$, at true strobe SKR maxima field component $i$ has a maximum at azimuth $\varphi_{B_{i}}^{*} \max$ given to modulo $360^{\circ}$ by

$$
\varphi_{B_{i}}^{*} \max =-\psi_{i}^{*}
$$

while in correspondence with equation $(5 c)$, at true strobe SKR maxima the rotating upward field-aligned current region is centered near azimuth $\varphi_{u F A C \max }^{*}$ given by

$$
\varphi_{u F A C \max }^{*} \approx-\psi_{r}^{*} \pm 90^{\circ},
$$

where again the upper sign corresponds to the southern system and the lower to the northern. For consistency with the above picture we then expect that $\varphi_{u F A C \max }^{*} \approx \varphi_{S K R} \simeq 296^{\circ}$, such that the true strobe SKR maxima occur when the rotating upward current region is colocated with the principal SKR sources in the postdawn sector. The implied values of $\psi_{r}^{*}$ are $\sim 154^{\circ}$ for the southern system, and $\sim 26^{\circ}$ (or equivalently $\sim 334^{\circ}$ ) for the northern, corresponding to the equatorial perturbation fields pointing approximately tailward and sunward, respectively, in agreement with previous determinations discussed in section 1 .

\section{Determination of Magnetic Phase Values}

[25] We now revisit the magnetic oscillation phases determined relative to the observed SKR modulation phases $\Phi_{S K R n}^{\prime}(t)$ and $\Phi_{S K R s}^{\prime}(t)$ for the northern (" $n$ ") and southern (" $s$ ") hemispheres respectively, as derived previously by Andrews et al. [2008, 2010b] and Provan et al. [2009a], and compare the long-term phase drifts found in these data with 


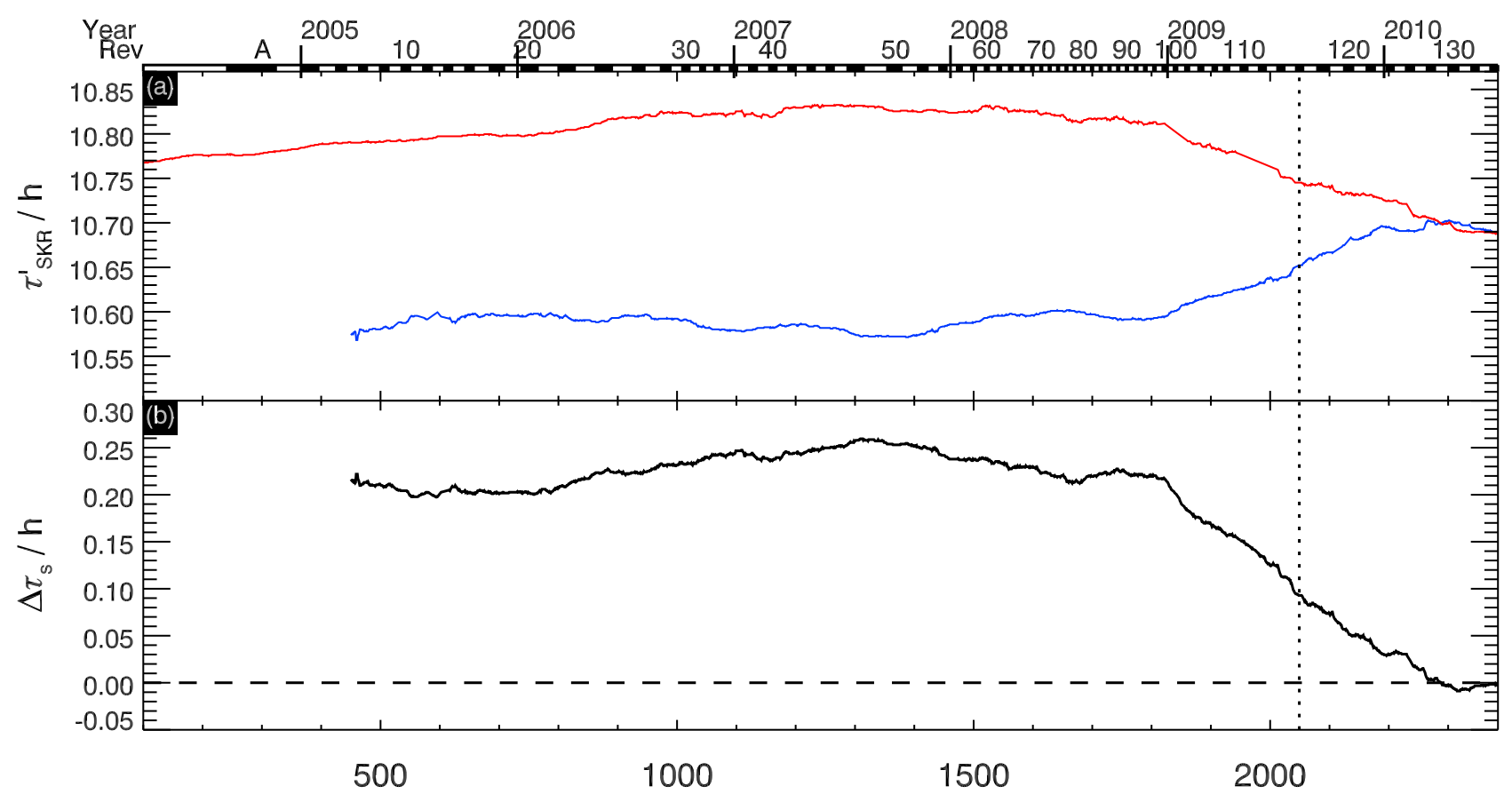

$\mathrm{t} /$ days

Figure 4. Time series plots of (a) the modulation period of the southern (red) and northern (blue) SKR emissions determined by Lamy [2011] and (b) their difference (south minus north). The vertical black dotted line indicates the time of Saturn vernal equinox. Other formatting follows that of Figure 3.

expectations based on equation (5e) and the positiondependent SKR phase deviations modeled in section 2 . We begin by briefly reviewing the derivation of the northern and southern SKR modulation phases, and then consider the relative phases $\psi_{i}^{\prime}$ of the magnetic field oscillations so determined.

\subsection{Derivation of SKR Modulation Phases}

[26] The northern and southern SKR modulation phases employed here are those derived by Lamy [2011], representing a modest revision and temporal extension of the numerical phase values employed previously by Andrews et al. [2010b] and Provan et al. [2011]. Modulation periods $\tau_{S K R ~}^{\prime}{ }_{n, S}(t)$ for the northern and southern SKR emissions, integrated over the SKR frequency band $40-500 \mathrm{kHz}$, were determined by Lomb-Scargle analysis using a 200 day sliding window and a time resolution of 1 day (see Lamy [2011] for further details). The influence of variations in the SKR period occurring on shorter time scales, such as those found by Zarka et al. [2007] to be correlated with changes in solar wind speed on the solar rotation period, are therefore removed. These periods are shown versus time in Figure $4 \mathrm{a}$, where the red line corresponds to the southern hemisphere, the longer period over most of the interval, and the blue line to the northern hemisphere. The difference between the southern and northern periods is shown in Figure $4 \mathrm{~b}$. It can be seen that the two values are separated by $\sim 0.2 \mathrm{~h}$ over most of the interval, but following shallow opposite extrema near $t \approx 1300$ days (mid-2007), the periods then converge to an essentially common value after $t \approx 2250$ days (early 2010). This con- vergence occurs $\sim 240$ days after vernal equinox, again indicated by the vertical dotted line in the plots. Similar results from a related analysis of SKR modulations have also been reported by Gurnett et al. [2010b].

[27] The observed SKR phase in each hemisphere is then determined by interpolating the resulting time series, and numerically integrating the values to give

$$
\Phi_{S K R n, s}^{\prime}(t)=360 \int_{0}^{t} \frac{d t^{\prime \prime}}{\tau_{S K R n, s}^{\prime}\left(t^{\prime \prime}\right)}+\Phi_{0 n, s} .
$$

Here $\Phi_{0 n, s}$ is a constant determined (to modulo $360^{\circ}$ ) by requiring SKR power maxima to correspond to phases of $360 \mathrm{~N}$ deg as in section 2.1 above.

\subsection{Derivation of Magnetic Oscillation Phases}

[28] Magnetic oscillation phases are derived from $1 \mathrm{~min}$ averaged spherical polar field components measured by the Cassini fluxgate magnetometer [Dougherty et al., 2004]. These data are processed by first subtracting Saturn's internal magnetic field using the "Cassini SOI" model of Dougherty et al. [2005], though use of any the existing empirical models would produce closely similar results. We note that in common with these models, the Cassini SOI model is exactly axisymmetric with zero azimuthal component, such that the azimuthal component of the residual field data remain as measured. These residual data are then band-pass filtered between 5 and $20 \mathrm{~h}$ using a standard Lanczos filter to isolate oscillations near the $\sim 11 \mathrm{~h}$ planetary period. The phases of 


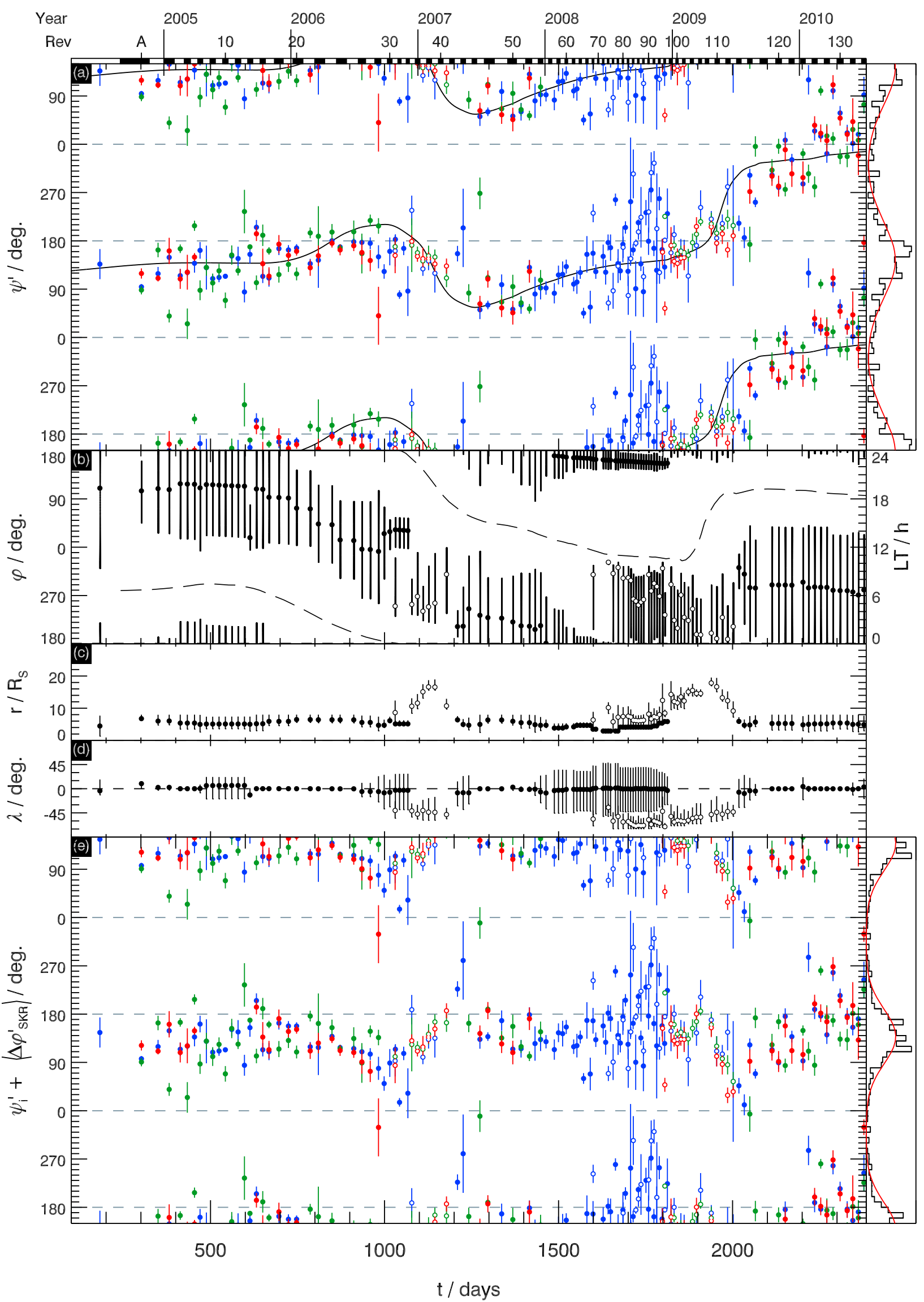

Figure 5

11 of 24 
the oscillations in each component $\psi_{i}^{\prime}$ relative to the observed SKR phases $\Phi_{S K R n, S}^{\prime}(t)$ are then determined (to modulo $360^{\circ}$ ) by least squares fitting the filtered residual data for selected data segments to the function given by equation (5a) with $B_{i 0}$ and $\psi_{i}^{\prime}$ as free parameters. Phase values are excluded from further analysis if (1) the resultant fits are of poor quality such that the fitted amplitude $B_{i}$ is less than twice the RMS "noise" in the data, (2) this amplitude is smaller than $0.2 \mathrm{nT}$, or (3) the quasi-static field in the $r$ or $\theta$ components generated by Saturn's ring current varies along the spacecraft trajectory on time scales similar to the field oscillations thus producing power in the filter band unrelated to the magnetospheric oscillations. Application of the latter criterion follows that in previous related studies, discussed in detail by Andrews et al. [2008, 2010b]. The phase fits rejected via these three criteria are $3 \%, 8 \%$, and $30 \%$, respectively. The total rejection rate is thus $41 \%$, the largest contributor by far being that associated with the ring current, which effects the $r$ or $\theta$ component data only.

[29] The selected data segments, usually intervals of one to two days containing two or more oscillation cycles, correspond to the inner near-equatorial quasi-dipolar magnetosphere within dipole $L \leq 8$ referred to as the "core" magnetosphere (a limit chosen to avoid the magnetic effects of quasi-static auroral field-aligned currents in the azimuthal field data [see Andrews et al., 2010b]), and to high-latitude open polar field lines in the north and south within radial distances of $25 \mathrm{R}_{\mathrm{S}}$ where warm and hot electron fluxes are continuously near instrument background [see Provan et al., 2009a; Andrews et al., 2010b]. These high-latitude intervals are also screened to ensure that no magnetosheath or solar wind data intervals are included. On orbits during the second highlatitude mission phase in 2008 (see Figure 3), however, we note that the spacecraft passed through the quasi-dipolar core on time scales shorter than the "planetary" period, such that phases can be determined only from unfiltered residual field data in these cases (Revs 55 to 97). However, the $r$ and $\theta$ components on these orbits are again compromised by the fields of the ring current, such that phases can be determined only for the azimuthal field component.
[30] The results of Andrews et al. [2010b] show that the magnetic field phases on northern and southern polar field lines are organized by the northern and southern SKR periods, respectively. Those within the core are also found to be organized by the southern SKR period over the interval from SOI to $t=2057$ days (to Rev 116 in mid-2009) which they studied, though with significant $\sim \pm 25^{\circ}$ Rev-to-Rev phase jitter due to the superposition of northern period oscillations of smaller amplitude [Provan et al., 2011], which contributes significantly to the noise in these data. In the following sections we thus consider magnetic field phase drifts in the core and southern polar field data combined, and separately in the northern polar field data.

\subsection{Southern Period Magnetic Phase Data}

[31] Magnetic oscillation phase data for the core region (solid dots) and southern polar region (open dots) relative to the observed phase of the southern SKR modulation are shown versus time in Figure 5a, over the same interval as Figures 3 and 4. Again, two cycles of phase are shown to clearly display the continuity and banding of the data. The phases of the $r$ (red) and $\theta$ (green) components are shown as measured, while $90^{\circ}$ has been subtracted from the phase of the $\varphi$ (blue) component in the core region and $90^{\circ}$ added in the polar region due to the opposite quadrature nature of these oscillations (Figure 1), to bring them to a common value. Vertical bars give an estimate of the relative uncertainty in these measurements, obtained by determining the range of phase required to produce a significant $10 \%$ increase in the RMS deviation of the model fit to the field data. We note that these phase values are essentially identical to those of Andrews et al. [2010b], except for slight differences due to the minor modifications in the numerical SKR phase model mentioned in section 3.1, and a temporal extension of the data set from Rev 116 to 134, now newly including Revs with apoapsis in the postdusk sector.

[32] Information on the location of the spacecraft during each data segment used to determine these phases is shown in Figures $5 \mathrm{~b}-5 \mathrm{~d}$, where the dots show the center times of these intervals, solid for the core region and open for the

\begin{abstract}
Figure 5. Plots showing southern period magnetic field phase data and related parameters. (a) Magnetic field phases relative to the observed southern hemisphere SKR modulation phase, determined in both the quasi-dipolar "core" region defined by dipole $L \leq 8$ (solid circles) and in the southern polar region (open circles), with red, green, and blue color coding for the phases of the $r, \theta$, and $\varphi$ field components, respectively. The phases of the $r$ and $\theta$ components are shown as measured, while $90^{\circ}$ has been subtracted from the phases of the $\varphi$ component determined in the core region, and $90^{\circ}$ added to those determined in the open field region, in order to account for the relative quadrature polarization of these oscillations. Vertical bars on each symbol give an estimate of the uncertainties determined by the range of phase required to produce a $10 \%$ increase in the RMS deviation of the model fit to the field data, and each point is plotted twice over the two cycles of phase shown. The solid black line shows the model phase drift due to the position-dependent SKR modulation phase deviation $\psi^{\prime}+\left\langle\Delta \varphi_{S K R}^{\prime}\right\rangle$ computed from equation (5e). The histogram to the right of Figure 5a shows the occupancy of $10^{\circ}$ intervals of phase normalized to the peak value (black), together with a wrapped normal distribution (red line) having the same directional mean and circular standard deviation (see Appendix A) as the phase data. Calendar year boundaries and Cassini Rev numbers are indicated at the top of the plot in the same manner as in Figure 3. (b) Vertical bars then show the range of azimuths traversed by the spacecraft during each interval for which phases are determined, with circles showing the position at the midpoint, the latter again being solid or open for core and polar data, respectively. The 200 day directional running average of the spacecraft azimuth is also shown by the dashed line, essentially reflecting the LT of spacecraft apoapsis (an equivalent LT scale is shown on the right of the plot). The radial (c) distance and (d) latitude of the spacecraft during each data interval are show in a similar manner. (e) The same phase data as in Figure 5a, adjusted by the addition of the modeled average phase deviation $\left\langle\Delta \varphi_{S K R}^{\prime}\right\rangle$, with the corresponding histogram and wrapped normal distribution again plotted to the right.
\end{abstract}


southern polar region. The dashed line in Figure $5 \mathrm{~b}$ shows the 200 day directional running average of the spacecraft azimuth, mainly reflecting the azimuth of apoapsis as shown in Figure 3c, while the vertical lines show the range of azimuths covered by the phase determinations, generally located at antipodal values near periapsis. Figures $5 \mathrm{c}$ and $5 \mathrm{~d}$ similarly show the radial and latitudinal ranges corresponding to the phase determinations, respectively.

[33] As previously discussed by Andrews et al. [2010b], the principal feature of the phase data is their distinct banding in value, though with significant scatter to which the jitter effect due to the presence of weaker northern period oscillations in the core data is a significant contributor. This banding shows that the magnetic oscillation phases are strongly organized by the southern SKR modulation phase, such that these phenomena have a nearly common period, within a few seconds overall. We note in particular that the data remain banded with similar relative polarization in the three field components in the postequinox data newly shown here, such that there is no evidence in these data for dominance of northern period oscillations in the core data during this interval. Northern dominance would produce highly scattered phase data relative to the southern period phase over intervals where the two periods are significant different. We thus take this overall data set to define the relative phase between the southern period field oscillations and the southern hemisphere SKR power modulations over the whole 6 year interval shown.

[34] As in equation (4), the directional mean $\bar{\psi}$ and the mean resultant length $\overline{R_{\psi}}$ of the phase values are given by

$$
\frac{1}{K} \sum_{k=1}^{K} e^{j \psi_{k}}=\overline{R_{\psi}} e^{\bar{\psi}},
$$

where we have now dropped component subscript $i$ since all the phase data are combined together having taken account of the relative polarization of the oscillations in the three field components ( $\psi$ corresponds directly to the phase of the $r$ and $\theta$ components). The directional mean phase value $\bar{\psi}$ so determined is $144^{\circ}$, which from equation (5b) indicates that at observed southern SKR maxima the radial field component has a maximum at azimuth $-144^{\circ}$, equivalent to $+216^{\circ}$, at a LT of $2.4 \mathrm{~h}$ in the postmidnight sector. This then is the direction of the southern period quasi-uniform equatorial field and the southern effective polar dipole at these times, both being in phase with the $r$ component, as previously indicated in section 1 (Figure 1). From equation (5c) we also note that the center of the associated upward field-aligned current in the southern ionosphere is then located at an azimuth of $+306^{\circ}$, i.e., a LT of $8.4 \mathrm{~h}$ in the postdusk center, essentially colocated with the peak in the SKR intensity shown in Figure 2 in accordance with expectation.

[35] The variance $V$ of the data about the directional mean, the latter being the phase value about which the data variance is a minimum, is given by

$$
V=1-\overline{R_{\psi}}
$$

(see Appendix A). For tightly grouped data, $V$ is approximately half the mean squared deviation of the phase values (in radians) about the directional mean. Equivalently, the circular standard deviation $\sigma_{\psi}$ of the data about the directional mean (in radians), employed here as a useful summary statistic, is given by

$$
\sigma_{\psi}^{2}=\ln \left(\frac{1}{\left(\overline{R_{\psi}}\right)^{2}}\right)
$$

(see Appendix A). The circular standard deviation $\sigma_{\psi}$ so determined for the data in Figure 5a is $71^{\circ}$. To the right of Figure 5a, we show a histogram of the phase values grouped into $10^{\circ}$ bins and normalized to the peak value, together with a "wrapped normal" distribution overplotted in red having this directional mean and circular standard deviation. The wrapped normal distribution is the analog for circular data of the "normal" (Gaussian) distribution for linear data [see, e.g., Mardia and Jupp, 2000] (see also Appendix A).

[36] As further discussed by Andrews et al. [2010b], however, long-term drifts are also present in these phase data, specifically slow drifts to larger phase between SOI and Rev 30 and between Revs 45 and 116, joined between by a shorter interval of more rapid drift to lower phase values. Direct interpretation of the positive phase gradients implies magnetic oscillation periods that are $\sim 10 \mathrm{~s}$ longer than the southern SKR modulation period during these intervals, while the intervening negative phase gradient implies a period that is $\sim 40 \mathrm{~s}$ shorter. In addition, as anticipated in the above discussion, we now also see a substantial shift in the phase values from Rev 117 to the end of the interval at Rev 134, these representing the first extended set of orbits with apoapsis in the postdusk sector. The directional mean phase of the earlier phase data (to end of Rev 116 at $t=2057$ days) is $150^{\circ}$ with a circular standard deviation of $53^{\circ}$, similar to the overall value, such that the equatorial quasi-uniform field and effective polar dipole point toward the postmidnight sector $(2.0 \mathrm{~h} \mathrm{LT})$ at southern SKR maxima as just discussed. However, the directional mean of the later data (beginning of Rev 117 to the end of the interval) is $357^{\circ}$ with a circular standard deviation of $60^{\circ}$, which from equation $(5 b)$ means that the equatorial quasi-uniform field and effective southern dipole now point toward azimuth $+3^{\circ}(12.2 \mathrm{~h} \mathrm{LT})$ in the immediate postnoon sector at times of observed southern SKR maxima during this epoch. Thus from equation $(5 \mathrm{c})$ the rotating upward field-aligned current region in the southern hemisphere is now centered at an azimuth of $\sim 93^{\circ}$ in the immediate postdusk sector at these times, consistent with the expected response of SKR sources located in the dusk sector which the spacecraft will then be observing, rather than the most intense sources in the midmorning sector observed earlier in the mission.

[37] We thus consider whether the magnetic field phase variations in Figure 5 are due to the position-dependent SKR modulation phase deviations discussed in section 2 . The apparent magnetic oscillation phase drifts expected to result from the SKR phase deviations are given by equation (5e), i.e., $\psi^{\prime}=\psi^{*}-\left\langle\Delta \varphi_{S K R}^{\prime}\right\rangle$, the position-dependent phase deviation $\Delta \varphi_{S K R}^{\prime}$ being suitably averaged to mimic the expected effect within the empirical SKR phase data and $\psi^{*}$ a constant to be determined. Suitable averages of $\Delta \varphi_{S K R}^{\prime}$ are obtained by interpolating the orbit-averaged values shown in Figure 3 at 1 min resolution, with a 200 day 
directional running average then being calculated to match the 200 day sliding window employed in the analysis of the SKR data. The best fit value of $\psi^{*}$ was then determined by minimizing the variance about zero of the difference between the data $\psi_{k}^{\prime}$ and the model $\Delta \psi_{k}^{\prime}=\psi_{k}^{\prime}-\left(\psi^{*}-\left\langle\Delta \varphi_{S K R}^{\prime}{ }_{k}\right\rangle\right)=\left(\psi_{k}^{\prime}+\left\langle\Delta \varphi_{S K R}^{\prime}{ }_{k}\right\rangle\right)-\psi^{*}$, where $k$ is the index of the phase data values determined during each Rev as in equation (7a). It is shown in Appendix A that this variance is minimized when $\psi^{*}=\overline{\left(\psi^{\prime}+\left\langle\Delta \varphi_{S K R}^{\prime}\right\rangle\right)}$, i.e., when $\psi^{*}$ is equal to the directional mean of the phase sum $\left(\psi_{k}^{\prime}+\left\langle\Delta \varphi_{S K R}^{\prime} k\right\rangle\right)$. The best fit value of $\psi^{*}$ so determined is $138^{\circ}$, and the resultant fitted curve is shown by the solid black line in Figure 5a.

[38] These results demonstrate that the phase drifts observed in the core and southern polar field data can be approximately accounted for in both form and magnitude by the positiondependent SKR phase deviations. The curve reproduces the slow drift to larger phase values observed prior to Rev 30 and after Rev 45, and the interval of more rapid fall between. It also reproduces the rapid increase in phase from $\sim 160^{\circ}$ to $\sim 320^{\circ}$ that accompanies the transfer of apoapsis into the postdusk sector after Rev 110. In Figure 5e we then show the same magnetic phase data as in Figure 5a "corrected" by the addition of the averaged SKR phase deviation $\left\langle\Delta \varphi_{S K R}^{\prime}\right\rangle$ i.e., the phase values $\psi^{\prime}+\left\langle\Delta \varphi_{S K R}^{\prime}\right\rangle$ (equation (5e)), thus representing the magnetic phase relative to the southern SKR true strobe phase given by equation $(3 \mathrm{~d})$. Little remains in these data of the secular phase drifts evident in Figure 5a, though the behavior particularly in the regions of rapid predicted phase change is not perfectly reproduced. The directional mean and standard deviation of these corrected data are $138^{\circ}$ and $51^{\circ}$, respectively, compared with $144^{\circ}$ and $71^{\circ}$ for the original data set in Figure 5a. The decrease in standard deviation from $71^{\circ}$ to $51^{\circ}$ is highly significant, as is clear from the histogram and overplotted wrapped normal curves to the right of Figures $5 \mathrm{a}$ and $5 \mathrm{e}$. The data are clearly more tightly grouped in Figure 5e than in Figure 5a. We also note from equations $(5 \mathrm{f})$ and $(5 \mathrm{~g})$ that the value $\psi^{*} \simeq 138^{\circ}$ corresponds to $\varphi_{B_{r}}^{*} \max \simeq-138^{\circ}=222^{\circ}(2.8 \mathrm{~h} \mathrm{LT})$ and $\varphi_{u F A C \max }^{*} \approx 312^{\circ}(8.8 \mathrm{~h} \mathrm{LT})$, the latter being close to the effective azimuth of the true strobe emission $\varphi_{S K R} \simeq 296^{\circ}(7.7 \mathrm{~h} \mathrm{LT})$ determined in section 2.1. Thus at the time of true strobe SKR maxima the southern period quasi-uniform equatorial field and effective polar dipole point consistently toward $2.8 \mathrm{~h} \mathrm{LT}$ in the postmidnight sector $( \pm 3.4 \mathrm{~h} \mathrm{LT})$, while the upward field-aligned current region is centered within one hour LT of the effective center of the most intense SKR sources in the midmorning sector (Figure 2a).

[39] These results imply that the small differences in period mentioned above and discussed previously by Andrews et al. [2008, 2010b] and Provan et al. [2009a] are in fact due to the restricted view of the SKR emissions influencing the SKR modulation phase rather than to actual differences in period. However, significant $\pm 51^{\circ}$ scatter about the mean value remains, a significant part of which represents the phase jitter effect in the core data mentioned above, together with the increased variability of the phases obtained from unfiltered azimuthal field data during the second interval of highlatitude orbits in 2008 as mentioned in section 3.2. In addition, inadequacies in the simple SKR seeing function employed here may also play a role, particularly with regard to observations at higher latitudes. Overall, however, a maximum possible $\sim 90^{\circ}$ phase shift between the field oscillations and the southern period SKR modulations over the whole 6 year interval investigated here, as suggested by the confined band of phase data in Figure 5e, implies an averaged period which is common to within $\sim 2 \mathrm{~s}$ (within $0.005 \%$ ). The implied variation of the common period over the interval will be discussed further in section 4.

\subsection{Northern Period Magnetic Phase Data}

[40] The SKR modulation phase deviation model derived in section 2 should in principle apply equally to the northern hemisphere data as to the southern, thus leading to related drifts in the relative phase of the northern period field oscillations. However, as indicated above, determinations of the phase of the northern period field oscillations are restricted to observations on northern open field lines, and are thus available only during the two limited intervals of highlatitude orbits indicated in Figure 3. Results are shown in Figure 6 in a similar format to that of Figures $5 \mathrm{a}-5 \mathrm{~d}$. The phase values relative to the observed northern SKR modulations shown in Figure $6 a$ have again been adjusted to account for the polarization of the oscillations. Specifically, while the $r$ component phase is again shown as measured, $90^{\circ}$ has been added to the $\varphi$ component, and $180^{\circ}$ to the $\theta$ component to bring them to a common value (see Figure 1). As noted previously by Andrews et al. [2010b], these phases are well organized by the northern SKR modulation phase, thus indicating a near-common period, but now about a directional mean value of $6^{\circ}$ with a circular standard deviation of $40^{\circ}$. From equation (5b) we note that this mean phase corresponds to the northern hemisphere effective polar dipole pointing toward $-6^{\circ}\left(+354^{\circ}\right)$ azimuth in the immediate prenoon sector (11.6 h LT) at the times of northern SKR maxima, as indicated in section 1 . Thus from equation $(5 \mathrm{c})$ the upward field-aligned currents in the northern hemisphere are centered at azimuth $264^{\circ}(5.6 \mathrm{~h} \mathrm{LT})$ at these times during the interval covered by the data, thus being located in the immediate predawn sector $\sim 2 \mathrm{~h} \mathrm{LT}$ earlier than the most intense SKR sources shown in Figure 2, but still in reasonable accord with expectation.

[41] Within the limited data intervals in Figure 6 there is no consistent evidence for the presence of phase drifts of a similar nature to those observed in the southern period oscillations. The variation expected on the basis of our model is again shown by the overplotted fitted solid line, which while not being grossly inconsistent with the data, also shows little clear correspondence. We also note that the circular standard deviation of the corrected data $\psi^{\prime}+\left\langle\Delta \varphi_{S K R}^{\prime}\right\rangle$ (not shown here) is $56^{\circ}$, significantly larger than the initial $40^{\circ}$ in the measured phases. Inadequacy of our simple seeing function as applied to higher-latitude data is one possible contributor to the lack of correspondence found here, this meriting future study beyond that of the present paper.

\section{Revised Period of the Southern Hemisphere Oscillations}

[42] The results in section 3 indicate that due to the dependence of the southern SKR modulation phase determinations on spacecraft position, usually but not always modest in magnitude, the southern periods determined from these data 


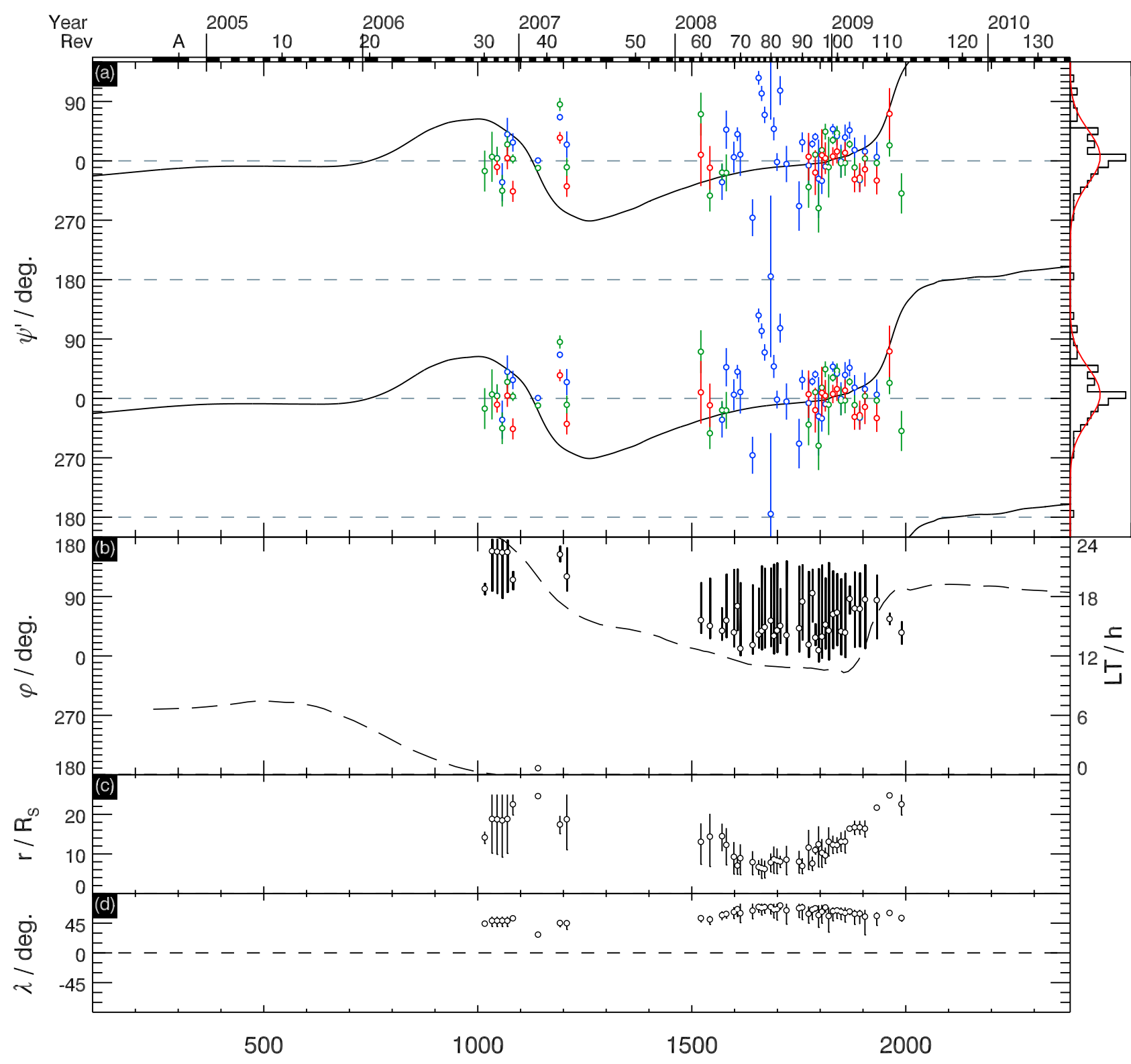

\section{$\mathrm{t} /$ days}

Figure 6. Northern period magnetic field phase data and related orbit parameters, in a format similar to that of Figures $5 \mathrm{a}-5 \mathrm{~d}$. The phases of the $r$ field component are again shown as measured, while those of the $\theta$ and $\varphi$ components have been adjusted by the addition of $180^{\circ}$ and $90^{\circ}$, respectively, to bring them to a common value.

deviate modestly from the true values, as revealed by the slow drifts in relative phase of the magnetic field oscillations. No evidence exists for such deviations in the much more sparsely observed northern period field data, however. In this section we therefore first estimate the true strobe period of the southern oscillations based on the model fits to the magnetic phase data in section 3 , and thus quantify the deviations from the period based on the observed SKR modulations alone. We then present an independent determination of the southern hemisphere period based on magnetic phase data alone, and compare the two data sets.

[43] The true strobe period of the southern SKR modulations, based on the true strobe phase $\Phi_{S K R}^{*}(t)$ in equation (3d), is related to the observed SKR period $\tau_{S K R}^{\prime}(t)$ determined by Lamy [2011] by

$$
\begin{aligned}
\tau_{S K R S}^{*}(t) & =\frac{360}{\frac{d}{d t}\left(\Phi_{S K R s}^{*}\right)}=\frac{360}{\frac{d}{d t}\left(\Phi_{S K R s}^{\prime}+\left\langle\Delta \varphi_{S K R}^{\prime}\right\rangle\right)} \\
& =\frac{\tau_{S K R s}^{\prime}}{1+\frac{\tau_{S K R S}}{360} \frac{d}{d t}\left(\left\langle\Delta \varphi_{S K R}^{\prime}\right\rangle\right)}
\end{aligned}
$$

where the angles are again in degrees. Results are displayed in Figure 7, where the black line in Figure 7a shows the observed southern SKR period $\tau_{S K R}^{\prime} s(t)$ determined as 


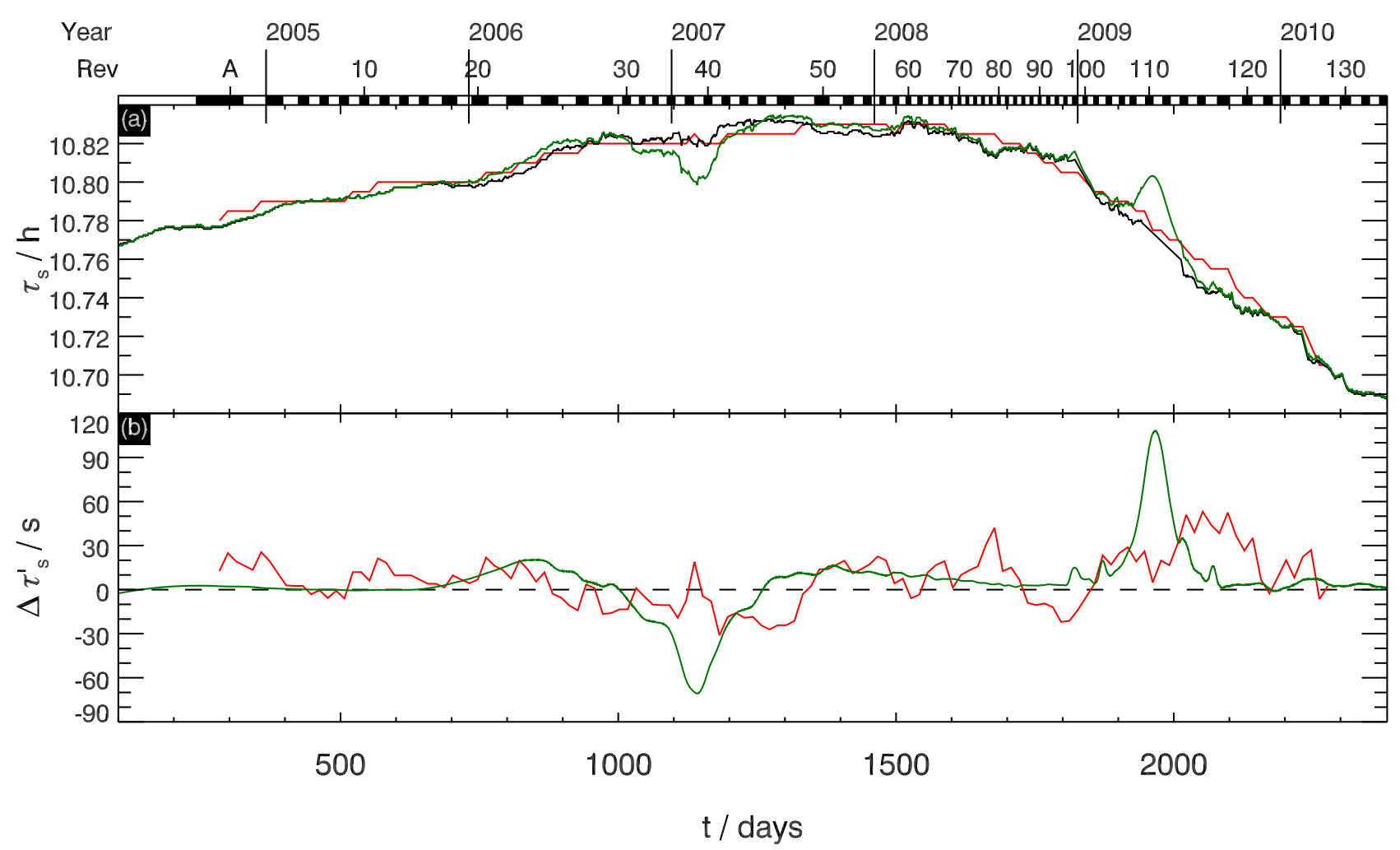

Figure 7. Plots comparing differing measures of the period of the southern SKR modulation and magnetic field oscillations. (a) The periods of the observed southern SKR modulation $\tau_{S K R s}^{\prime}(t)$ determined by Lamy [2011] (black line), the SKR true strobe period "corrected" for modeled position-dependent phase deviations $\tau_{S K R S}^{*}(t)$ calculated from equation (8) (green line), and the independently determined magnetic field period $\tau_{M s}(t)$ (red line) obtained from the continuous peak in the periodogram shown in Figure 8a. (b) The difference between the true strobe and observed SKR periods $\tau_{S K R s}^{*}-\tau_{S K R s}^{\prime}$ (green line) and between the magnetic field period and the observed SKR period $\tau_{M s}-\tau_{S K R s}^{\prime}$ (red line).

described in section 3.1 , as shown previously by the red line in Figure 4a. The green line in Figure 7 then shows the modified period $\tau_{S K R}^{*}(t)$ given by equation (8), obtained by numerical differentiation of the averaged $\Delta \varphi_{S K R}^{\prime}$ values. It can first be seen that the deviations about $\tau_{S K R}^{\prime}(t)$ are usually small, except for intervals near $t=1150$ and 1950 days which correspond to sharp changes in $\left\langle\Delta \varphi_{S K R}^{\prime}\right\rangle$ (Figure 5) due to rapid evolution of the spacecraft orbit (Figure 3 ). For better clarity in Figure $7 \mathrm{~b}$ we thus show the difference $\Delta \tau_{s}^{\prime}=\tau_{S K R s}^{*}-\tau_{S K R}^{\prime} s$ between these periods (green line). The overall values are small, $\sim 10 \mathrm{~s}$ or less, except near $t=1150$ and 1950 days as noted above where opposite extrema peaking at $\sim 70$ and $\sim+110$ s occur. While we do not ascribe significance to the detailed values in these regions, the integrated effect of these differences are just such as to produce the overall negative and positive changes in phase by up to $\sim 180^{\circ}$ evident in the magnetic phase values in Figure 5.

[44] The southern oscillation true strobe period shown by the green lines in Figure 7 is based initially on southern hemisphere SKR data, corrected for the modeled positiondependent SKR phase drift. However, it is also possible to use the southern period magnetic field data directly to estimate the variation of the oscillation period. In essence this can be done by computing the magnetic field phases within sliding subintervals of the data using guide phases of fixed period, and determining the period for which these phases are most nearly constant. For practical calculation, the phases of the magnetic oscillations are first determined using the same least squares method as described in section 3.2, but relative to a guide phase $\Phi_{0}(t)$ corresponding to a fixed period of exactly $\tau_{0}=10.82 \mathrm{~h}$ (the period near the center of the interval) given simply by

$$
\Phi_{0}(t)=\frac{360 t}{\tau_{0}} \operatorname{deg}
$$

where $t$ is again time since 00 UT on 1 January 2004, and we note that $\Phi_{0}(0) \equiv 0^{\circ}$. If the field oscillation phase so determined for a given field component on a given Rev is $\psi_{0}$, then for any other close period $\tau$, the phase is modified to

$$
\psi=\psi_{0}-360 t\left(\frac{1}{\tau}-\frac{1}{\tau_{0}}\right) \operatorname{deg} .
$$

For a given subinterval containing $N$ phase measurements from all field components (adjusting the phase of the quadrature $\varphi$ component to agree with that of the $r$ and $\theta$ components as above), the mean resultant length $\overline{R_{\psi}}$ of these data is calculated from equation (7a), and the value of $\tau$ determined which gives the maximum value of this quantity. We recall 


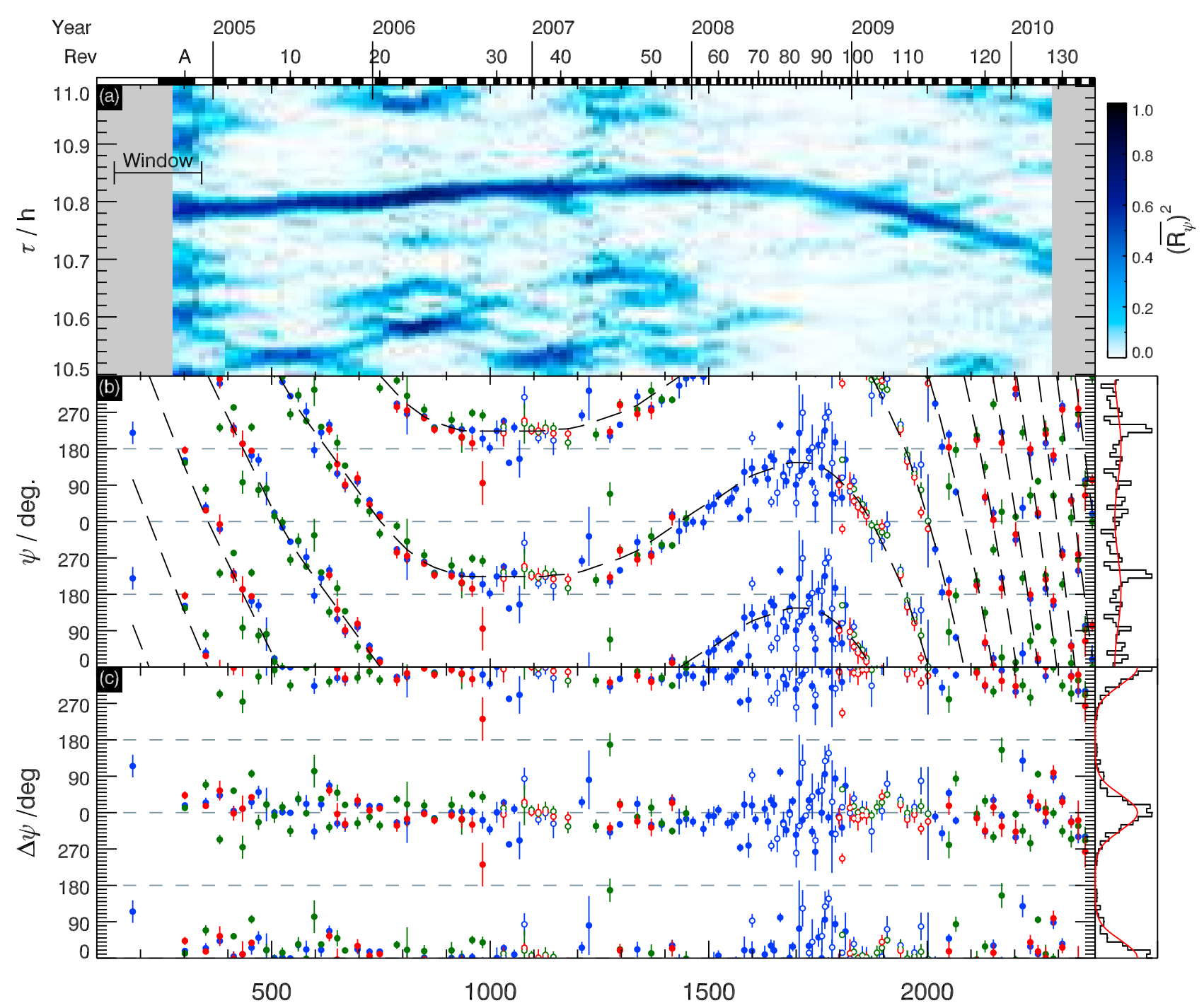

$\mathrm{t} /$ days

Figure 8. Plots relating to the determination of the southern rotation period from magnetic field data alone. (a) A color-coded periodogram of the squared mean resultant length $\left(\overline{R_{\psi}}\right)^{2}$ of the southern period magnetic phase data computed with a cadence of 15 days using a sliding window of 200 day length as indicated by the icon on the left of the figure. Oscillation period $\tau(\mathrm{h})$ is shown on the vertical axis versus time $t$ (days) on the horizontal axis. Grey regions at both ends of the plot indicate intervals for which values cannot be computed due to the 200 day window. Rev and calendar year boundaries are indicated at the top as in Figure 3. (b) Magnetic phases determined relative to the phase of an oscillation of fixed $10.82 \mathrm{~h}$ period, using the same intervals of field data as in Figure 5 and with the phase of the quadrature $\varphi$ field component adjusted as in Figure 5. The black dashed line shows the difference phase $\Phi_{0}(t)-\Phi_{M s}(t)$ between the phase of the fixed period oscillation $\Phi_{0}(t)$ and the magnetic phase $\Phi_{M s}(t)$ computed from the period corresponding to the continuous peak in the periodogram in Figure 8a. (c) The measured phases relative to the difference phase $\Phi_{0}(t)-\Phi_{M}(t)$ shown by the dashed line in Figure $8 \mathrm{~b}$.

from the discussion in section 3.3 (see also Appendix A) that $V=1-\overline{R_{\psi}}$ gives the variance of the data about the directional mean (the minimum variance about any phase value), such that maximizing $\overline{R_{\psi}}$ (between limits of 0 and 1 ) is equivalent to minimizing variance $V$. To best compare with the period determined from the southern SKR modulations, we use subinterval windows of 200 days, recomputed every 15 days, roughly equal to the average spacing between successive sets of phase measurements. Values of $\overline{R_{\psi}}$ were then computed for each subinterval for periods $\tau$ between 10.5 and $11.0 \mathrm{~h}$ in steps of $0.005 \mathrm{~h}(18 \mathrm{~s})$.

[45] In Figure 8a we show a color-coded plot of $\left(\overline{R_{\psi}}\right)^{2}$ versus oscillation period $\tau$ on the vertical axis and time $t$ on the horizontal axis. Gray vertical bands at the beginning and end of the interval indicate times where results cannot be shown due to the 200 day data window extending beyond 
the first or last phase data points available. It can be seen that a continuous maximum exists in these values near $\sim 10.8 \mathrm{~h}$ throughout the interval, which we take to correspond to the period of the southern hemisphere magnetic field oscillations. Secondary short-lived maxima also appear on either side of this main peak with displacements $\sim 0.2 \mathrm{~h}$. These are due to aliasing introduced in subintervals in which the spacing between successive phase determinations (Revs) is relatively constant. We note that this method unmodified is not expected to simultaneously reveal evidence of the northern oscillation period in Figure 8 despite the presence of such oscillations within the core region, and indeed none is evident in the periodogram. The magnetic field period given by the peak $\left(\overline{R_{\psi}}\right)^{2}$ value within the continuous band, $\tau_{M s}(t)$ is shown by the red lines in Figure 7, for comparison with the observed southern SKR period determined by Lamy [2011] (black) and the SKR true strobe period derived above (green). It can first be seen in Figure $7 \mathrm{a}$ that the magnetic field period is in close overall agreement with the SKR periods. The deviation in the magnetic field period from the observed SKR values shown in Figure $7 \mathrm{~b}$ (red line) again shows positive and negative values of typically a few tens of seconds, which generally agree in sense and magnitude with those deduced from the magnetic phase drift model in section 3 (green line). In particular we note the interval of mainly negative values centered near $t \approx 1150$ days, and the large positive values after $t \approx 1900$ days peaking at $\sim 50 \mathrm{~s}$, which represent somewhat smoothed versions of the larger peaks in the green line. A few intervals of modest apparent discrepancy also exist between the two periods, such as the intermittently larger positive values in the red compared with the green curve prior to $t \approx 600$ days.

[46] In Figures $8 \mathrm{~b}$ and $8 \mathrm{c}$ we demonstrate how this magnetic field period organizes the magnetic oscillation phases within the core region and on southern open field lines over this interval. In Figure $8 \mathrm{~b}$ we show the magnetic phase values determined relative to the guide phase of the oscillation whose period is exactly $10.82 \mathrm{~h}$ (equation (9a)) as described above, in a similar format to Figure 5 (the phases of the quadrature $\varphi$ components being adjusted to that of $r$ and $\theta$ as before). The black dashed line then shows the differenced oscillation phase $\Phi_{0}(t)-\Phi_{M s}(t)$, where $\Phi_{0}(t)$ is the guide phase of the $10.82 \mathrm{~h}$ oscillation given by equation (9a), and $\Phi_{M s}(t)$ is the phase of the southern period magnetic field oscillations obtained by numerically integrating the magnetic period versus time as for the SKR phase in equation (6). The constant in the latter integral, equal to $267^{\circ}$, has then been calculated by determining the best fit between the resulting curve and the data, obtained by the same method as used to fit the model curves to the phase data in Figures 5 and 6 . In this system the $r$ and $\theta$ component field oscillations are thus expressed as

$$
B_{i}(\varphi, t)=B_{i 0} \cos \left(\Phi_{M s}(t)-\varphi\right)
$$

with the $\varphi$ components being in lagging and leading quadrature in the equatorial and southern polar regions, respectively. At time $t$ the southern period quasi-uniform equatorial field and southern effective polar dipole thus point toward azimuth

$$
\varphi_{B_{r} \max }(t)=\Phi_{M s}(t)
$$

with the southern upward field-aligned current region being centered $\sim 90^{\circ}$ ahead in azimuth and the downward fieldaligned current region $\sim 90^{\circ}$ behind. Numerical values of the southern magnetic field period $\tau_{M}{ }_{s}(t)$ and corresponding integrated phase $\Phi_{M s}(t)$ are available in the auxiliary material. ${ }^{1}$ The difference phase shown in Figure $8 \mathrm{~b}$ decreases with time when the magnetic oscillation period is less than the guide period of $10.82 \mathrm{~h}$, increases with time when the oscillation period is greater than the guide period, and is constant (i.e., the difference phase has an extremum) when the oscillation period is just equal to the guide period. As expected, the overall fit to the phase data is excellent, though this is less obviously so after $t \approx 1900$ days, when the period of the field oscillations decreases significantly below the $10.82 \mathrm{~h}$ period of the guide phase such that the difference phase decreases rapidly with time.

[47] In Figure 8c we thus show the deviation $\Delta \psi=\psi-$ $\left(\Phi_{0}(t)-\Phi_{M s}(t)\right)$ of the phase data from the fitted line, equal to the deviation of the data from the phase of the model in equations (10a) and (10b). The data cluster about zero over the whole interval, with no indication of subintervals with secular change. The circular standard deviation of these data about the directional mean of zero is $43^{\circ}$, significantly lower than the $51^{\circ}$ circular standard deviation of the corrected magnetic phase data using the SKR phase deviation model shown in Figure 5e.

[48] We finally compare the three phase functions discussed here whose derivatives define measures of the slowly varying rotation period of the southern hemisphere oscillations. These are the observed phase of the southern SKR modulations $\Phi_{S K R}^{\prime}(t)$ derived from RPWS data by Lamy [2011], the true strobe phase of the southern SKR modulations $\Phi_{S K R}^{*} s(t)$ corrected for position-dependent SKR phase deviations according to the model derived in section 2 (equation (3d)), and the phase determined independently from the magnetic field oscillation data $\Phi_{M s}(t)$. The black line in Figure 9a shows the difference between the true strobe and observed SKR phases, $\left\langle\Delta \varphi_{S K R}^{\prime}\right\rangle=\Phi_{S K R s}^{*}-\Phi_{S K R s}^{\prime}$, which, apart from a constant, is the inverse of the black line in Figure 5a. Its value varies by several tens of degrees on either side of zero over much of the interval, before declining strongly after $t \approx 1900$ days as the spacecraft apoapsis moves into the postdusk sector. The red line in Figure 9a then shows the difference between the magnetic oscillation phase and the observed SKR phase, $\Delta \Phi_{M s}^{\prime}=\Phi_{M s}-\Phi_{S K R s}^{\prime}$. Overall, this curve closely mirrors the behavior of $\left\langle\Delta \varphi_{S K R}^{\prime}\right\rangle$ shown by the black line, apart from a near-constant offset of $\sim 220^{\circ}$. Substituting $\Phi_{M s}=\Delta \Phi_{M s}^{\prime}+\Phi_{S K R s}^{\prime}$ into equation (10a) shows that at observed SKR maxima $\left(\Phi_{S K R s}^{\prime}=360 N \mathrm{deg}\right)$ the $r$ component has a maximum at an azimuth $\varphi_{B_{r} \text { max }}=\Delta \Phi_{M s}^{\prime}$, such that this quantity also shows the azimuth of the quasiuniform equatorial field and effective southern polar dipole at observed southern SKR maxima. The value lies close to $\sim 220^{\circ}$ during the initial phase of the mission, before declining rapidly after $t \approx 1900$ days to near-zero values. The field direction at observed SKR maxima thus rotates from postmidnight to noon via dusk in the latter interval, in accordance with the discussion in section 3.3 , corresponding

\footnotetext{
${ }^{1}$ Auxiliary materials are available in the HTML. doi:10.1029/ 2011JA016636.
} 


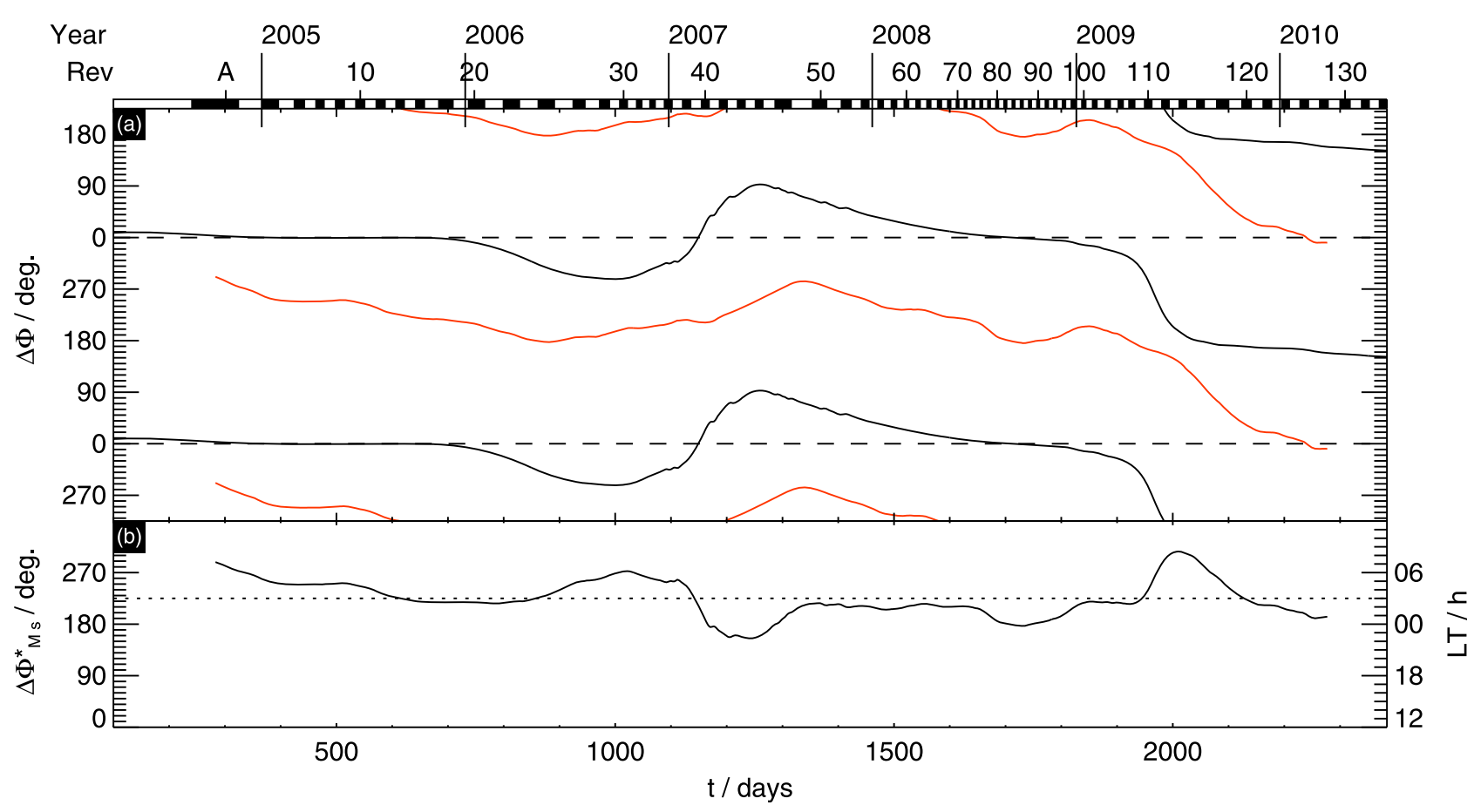

Figure 9. Time series plots of southern period SKR and magnetic oscillation phase differences. (a) The black line shows the difference between the true strobe and "observed" SKR phases, $\left\langle\Delta \varphi_{S K R}^{\prime}\right\rangle=\Phi_{S K R s}^{*}-\Phi_{S K R s}^{\prime}$, where $\left\langle\Delta \varphi_{S K R}^{\prime}\right\rangle$ corresponds to the averaged position-dependent SKR phase deviation illustrated in Figure 5a. The red line shows the difference between the independently determined magnetic oscillation phase and the observed SKR phase, $\Delta \Phi_{M s}^{\prime}=\Phi_{M s}-\Phi_{S K R s}^{\prime}$, equal to the azimuth of the quasi-uniform equatorial field and effective southern polar dipole at observed southern SKR maxima. The plot covers two complete cycles of phase, and Rev and calendar year boundaries are indicated on the top as in Figure 3. (b) The difference between the magnetic oscillation phase and the true strobe SKR phase, $\Delta \Phi_{M s}^{*}=\Phi_{M s}-\Phi_{S K R}^{*}$, which similarly shows the azimuth of the southern period field at true strobe SKR maxima. The horizontal dotted line shows the directional mean of these data, equal to $225^{\circ}$.

to a related rotation of the center of the southern upward field-aligned current region at these times from postdawn to dusk via midnight $\left(90^{\circ}\right.$ ahead in azimuth of the field direction).

[49] In Figure $9 \mathrm{~b}$ we then show the difference between the magnetic oscillation phase and the true strobe SKR phase, $\Delta \Phi_{M s}^{*}=\Phi_{M s}-\Phi_{S K R s}^{*}$, which similarly shows the azimuth of the southern period field at true strobe SKR maxima. This is now approximately constant with time as expected, with a directional mean value of $225^{\circ}$ (corresponding to $3.0 \mathrm{~h} \mathrm{LT}$ ), and a circular standard deviation of $32^{\circ}( \pm 2.1 \mathrm{~h} \mathrm{LT})$. The corresponding LT of the center of the southern upward fieldaligned current region is then at $9.0 \mathrm{~h}$ in the postdawn sector, one hour later than the effective azimuth of the midmorning true strobe sources.

\section{Summary}

[50] In this study we have examined the phase relationship between the planetary period modulations in the intensity of SKR emissions at Saturn and related oscillations in the magnetospheric magnetic field observed by the Cassini spacecraft over the 6 year interval from mid-2004 to mid2010. Specifically, we have examined the origin of the slow long-term phase drifts between these oscillations that have been reported in previous studies [Andrews et al., 2008, 2010b;
Provan et al., 2009a], which initially suggest the presence of differences in period of a few tens of seconds. Here we have examined the possibility that the SKR modulations are not strobe like as believed hitherto on the basis of Voyager observations, but instead rotate around the northern and southern auroral ovals in the same manner that the magnetic field and plasma oscillations rotate about the planet in the magnetosphere, possibly connected with a rotating system of field-aligned magnetosphere-ionosphere coupling currents. Initial evidence for such rotating modulation has recently been obtained by Lamy [2011] from directionresolved SKR emission data. Near-strobe-like behavior of the SKR modulation phase will still occur in a restricted LT sector from predawn to postnoon in which the most intense midmorning SKR sources are near continuously visible, but elsewhere the modulation phase will depend significantly on spacecraft LT due to conical beaming of the emission which severely restricts the view of SKR sources around the auroral oval, especially in the postdusk sector antipodal to the most intense SKR sources. Here we have modeled these effects and have examined whether they can account for the observed phase drifts between the SKR and magnetic field data. Our principal findings are summarized as follows.

[51] 1. Magnetic field phases have been determined relative to the north-south separated SKR phases derived by Lamy [2011] for both the quasi-dipolar core region (dipole 
$L \leq 8)$ and the two polar open field regions, extending the study of Andrews et al. [2010b] between SOI (July 2004) and Rev 116 (August 2009) to Rev 134 (July 2010). The newly encompassed near-equatorial postequinox data represent the first extended observations in which the spacecraft apoapsis lies in the postdusk sector, nearly antipodal to the most intense midmorning SKR sources. The relative phase of these oscillations is found to remain well organized by the southern SKR period over this interval, with a polarization as expected for southern period oscillations. The new data are thus taken to extend the near continuously determined phase of the southern period field oscillations observed in both the core and on southern polar field lines combined over six years of in-orbit observations, relative to the observed phase of the southern SKR emission modulations. Direct observations of northern period field oscillations remain confined to the north polar region observed during two high-latitude phases of the Cassini mission.

[52] 2. Results for the earlier interval of southern period magnetic field data remain similar to those determined using earlier SKR phase models, with slow drifts in relative phase, both positive and negative, of several tens of degrees over intervals of a few hundred days. On average, however, the relative phase is such that at southern SKR maxima the rotating quasi-uniform equatorial field and effective southern polar dipole point tailward and toward dawn, radially outward at $\sim 2-3 \mathrm{~h} \mathrm{LT}$. This is consistent with the upward field-aligned currents of the corresponding rotating southern current system being centered at $\sim 8-9$ h LT at southern SKR maxima, essentially colocated with the most intense SKR sources in the midmorning sector. However, following the change of spacecraft apoapsis to the postdusk sector in the first half of 2009 the relative phase of the field oscillations also changed by $\sim 180^{\circ}$, such that the direction of the quasiuniform equatorial field and effective southern polar dipole at observed SKR maxima rotated via dusk into the noon sector. The latter orientation implies that at the time of observed SKR maxima in these later data the southern upward field-aligned currents are instead centered in the dusk sector, approximately corresponding to the LT of apoapsis of the spacecraft. This effect is suggested to be due to the SKR observations then corresponding to sources in the dusk hemisphere whose combined modulation phase is in approximate antiphase with the most intense sources in the midmorning sector, as observed previously in auroral UV emissions [Nichols et al., 2010a]. The earlier smaller phase drifts are then suggested to correspond to a less extreme expression of this effect during intervals when the spacecraft apoapsis was almost continuously located in the midnight to predusk sector via dawn and noon, where the SKR modulation phase remains closer to the most intense sources in the midmorning sector.

[53] 3. A simple theoretical model of this effect has been constructed assuming that the rotating SKR emission modulations correspond to a fixed fraction of the LT-dependent SKR intensity determined by Lamy et al. [2009], and that due to the conical beaming of the SKR radiation, emission is detected only from two narrow azimuthal windows around the auroral oval located on either side of the spacecraft azimuth. Specifically, based on the results of Lamy et al. [2008b] and Cecconi et al. [2009] we assume "seeing windows" that are $15^{\circ}$ of azimuth ( $1 \mathrm{~h} \mathrm{LT}$ ) wide centered on azimuths $55^{\circ}(\sim 3.7 \mathrm{~h} \mathrm{LT})$ on either side of the spacecraft azimuth. Using this model, position-dependent SKR phase deviations have been computed relative to the true strobe SKR phase that would be detected by an observer with a complete view of all SKR sources in each hemisphere, which have then been averaged over sliding 200 day intervals to mimic the SKR phases determined by Lamy [2011]. It is found that these phase deviations can indeed account for the relative phase drifts observed in the southern period magnetic field data, both in sign and magnitude. After "correcting" the SKR phases using the model, it is found that the long-term phase drift effect in the magnetic field data is strongly reduced. At true strobe SKR maxima, corresponding to maxima of the most intense sources in the midmorning sector, the quasi-uniform equatorial field and effective southern polar dipole then point radially outward at $\sim 3 \pm 3 \mathrm{~h} \mathrm{LT}$ consistently throughout the 6 year interval.

[54] 4. The "correction" of the observed southern SKR modulation phase for position-dependent phase deviations necessarily also implies a related correction of the southern hemisphere SKR rotation period. These generally correspond to positive and negative deviations about the observed southern SKR period of a few tens of seconds over intervals of a few hundred days, though peaking at several tens of seconds during two intervals of rapid orbit evolution. While not ascribing significance to the details of these variations, overall they integrate over such intervals to give rise to the observed phase deviations in the magnetic field data. We have also for the first time obtained an independent determination of the southern rotation period and integrated phase directly from the core and southern polar magnetic field observations. These are found to be in good overall agreement with the true strobe values determined from the above model, showing similar deviations from the values obtained directly from the observed SKR modulations. Specifically, at true strobe SKR maxima the quasi-uniform equatorial field and effective southern polar dipole are found to point radially outward at $\sim 3 \pm 2 \mathrm{~h}$ LT consistently throughout the interval. Numerical values of the southern magnetic field period and integrated phase data are available in the auxiliary material.

[55] 5. Observations of northern period polar field oscillations remain restricted to two intervals of high-latitude Cassini data investigated previously by Provan et al. [2009a] and Andrews et al. [2010b], that occur prior to the rotation of the spacecraft apoapsis into the postdusk sector. These data show no evidence of the more modest phase drifts relative to the northern period SKR modulation phase expected during these intervals based on the theoretical model. This might be due to an inadequacy in the model SKR seeing function as applied to northern high-latitude radio observations, an aspect requiring further investigation. The relative phasing is then such that at northern SKR maxima the effective northern polar transverse dipole points consistently toward $\sim 11.5 \pm 2.5 \mathrm{~h} \mathrm{LT}$ in the immediate prenoon sector. This implies that at these times the northern upward field-aligned current region is centered in the immediate predawn sector, about two hours earlier than the LT of the most intense midmorning SKR sources determined by Lamy et al. [2009].

\section{Appendix A: Directional Statistics}

[56] In this appendix we briefly review the numerical techniques employed to analyze the phase data examined in 
this paper. Suppose we wish to obtain the mean of a set of somewhat scattered phase data, then simply calculating the arithmetic mean in the usual way is inappropriate, since the result depends on the arbitrary choice of the $360^{\circ}$ phase interval used to represent the data, e.g., whether $-180^{\circ}$ to $+180^{\circ}$ or $0^{\circ}$ to $360^{\circ}$. For data that are in effect defined on a circle rather than on a line, directional statistics must instead be employed [e.g., Mardia and Jupp, 2000].

[57] If we consider a set of $K$ angles $\theta_{k}$, then the directional mean of these angles $\bar{\theta}$ is given by

$$
\frac{1}{K} \sum_{k=1}^{K} e^{j \theta_{k}}=\bar{R} e^{j \bar{\theta}}
$$

where $\bar{R}$ is the modulus and $\bar{\theta}$ the argument of the complex sum. In terms of real functions, if we consider the trigonometric sums

$$
\bar{C}=\frac{1}{K} \sum_{k=1}^{K} \cos \left(\theta_{k}\right) \text { and } \bar{S}=\frac{1}{K} \sum_{k=1}^{K} \sin \left(\theta_{k}\right),
$$

then the directional mean is given by

$$
\bar{\theta}=\tan ^{-1}\left(\frac{\bar{S}}{\bar{C}}\right)
$$

where account is taken of the signs of both $\bar{S}$ and $\bar{C}$ individually such that the result is defined (to modulo $360^{\circ}$ ) over the full $360^{\circ}$ range. These formulas have been employed in all the averages of phase data in this paper, for example, to calculate the orbit-averaged value of $\Delta \varphi_{S K R}$ in section 2.1, and the mean of the magnetic oscillation phase values in section 3.3 (equations (4) and (7a)-(7c)). Parameter $\bar{R}$, called the mean resultant length, is given by

$$
\bar{R}=\sqrt{\bar{S}^{2}+\bar{C}^{2}},
$$

where $0 \leq \bar{R} \leq 1$. We note that the calculation of both $\bar{R}$ and $\bar{\theta}$ involves no selection of phase interval, arbitrary to modulo $-360^{\circ}$, on which to perform the calculation, and that the results are also independent of choice of origin for $\theta$. If the origin is changed such that $\theta \rightarrow \theta-\theta_{0}$, for example, then multiplying equation (A1a) by $e^{-i \theta_{0}}$ we have

$$
\frac{1}{K} \sum_{k=1}^{K} e^{i\left(\theta_{k}-\theta_{0}\right)}=\bar{R} e^{i\left(\bar{\theta}-\theta_{0}\right)},
$$

such that $\bar{R}$ is unchanged and $\bar{\theta} \rightarrow \bar{\theta}-\theta_{0}$. Further, multiplying equation (A1a) by $e^{-i \bar{\theta}}$ we have

$$
\frac{1}{K} \sum_{k=1}^{K} e^{i\left(\theta_{k}-\bar{\theta}\right)}=\bar{R}
$$

such that

$$
\frac{1}{K} \sum_{k=1}^{K} \cos \left(\theta_{k}-\bar{\theta}\right)=\bar{R} \text { and } \frac{1}{K} \sum_{k=1}^{K} \sin \left(\theta_{k}-\bar{\theta}\right)=0 .
$$

Parameter $\bar{R}$ describes the "concentration" of the angles $\theta_{k}$ about the directional mean $\bar{\theta}$, with $\bar{R} \rightarrow 1$, its maximum value, when all $\theta_{k} \rightarrow \bar{\theta}$, and $\bar{R} \rightarrow 0$, its minimum value, when for every $\theta_{k}$ there is a corresponding value $\theta_{k}+180^{\circ}$ in the data set. An appropriate measure of the "variance" $V_{\bar{\theta}}$ of the angles about the directional mean is

$$
V_{\bar{\theta}}=\frac{1}{K} \sum_{k=1}^{K}\left(1-\cos \left(\theta_{k}-\bar{\theta}\right)\right)=1-\bar{R}
$$

where we note that for small deviations of $\theta_{k}$ from $\bar{\theta}$ we have

$$
V_{\bar{\theta}} \simeq \frac{1}{2 K} \sum_{k=1}^{K}\left(\theta_{k}-\bar{\theta}\right)^{2},
$$

such that in this limit $V_{\bar{\theta}}$ is given by half the mean square deviation of the angles (in radians) from the directional mean. The variance about any other angle $\alpha$ is then given by

$$
V_{\alpha}=\frac{1}{K} \sum_{k=1}^{K}\left(1-\cos \left(\theta_{k}-\alpha\right)\right)=V_{\bar{\theta}}+2 \bar{R} \sin ^{2}\left(\frac{\bar{\theta}-\alpha}{2}\right),
$$

where use has been made of equation (A1f). From equation (A2c) we thus see that the directional mean $\bar{\theta}$ is the angle about which the variance of the values $\theta_{k}$ is a minimum, and that the minimum value of the variance is $V_{\bar{\theta}}=1-\bar{R}$

[58] Angular data can conveniently be described by a number of conventional population distributions. Here we choose to use the "wrapped normal distribution" [see, e.g., Mardia and Jupp, 2000], consisting in essence of a linear normal (Gaussian) distribution "wrapped" around a circle, given by

$$
f(\theta)=\frac{1}{\sigma \sqrt{2 \pi}} \sum_{n=-\infty}^{\infty} \exp \left[-\frac{(\theta-\bar{\theta}+2 \pi n)^{2}}{2 \sigma^{2}}\right],
$$

where $\bar{\theta}$ is the directional mean and $\sigma$ the circular standard deviation of the distribution. For this distribution it can be shown that

$$
\bar{R}=e^{-\sigma^{2} / 2} \text { so that } \sigma=\sqrt{\ln \left(\frac{1}{\bar{R}^{2}}\right)},
$$

where $\sigma$ is in radians. The circular standard deviation of a set of angular data derived using equations (A1d) and (A3b) then provides a useful summary statistic that is analogous to the usual standard deviation for data on a line, and has been so employed in discussing the scatter of the magnetic phase data in sections 3 and 4. In Figure A1 we illustrate the form of this distribution for $\bar{\theta}=180^{\circ}$ and various values of $\sigma$, in which we include terms in equation (A3a) for $-10 \geq n \geq 10$, providing more than adequate accuracy. These forms are those employed to fit the distributions of phase data e.g., in Figures 5, 6, and 8, where they are plotted with the same number of terms. 


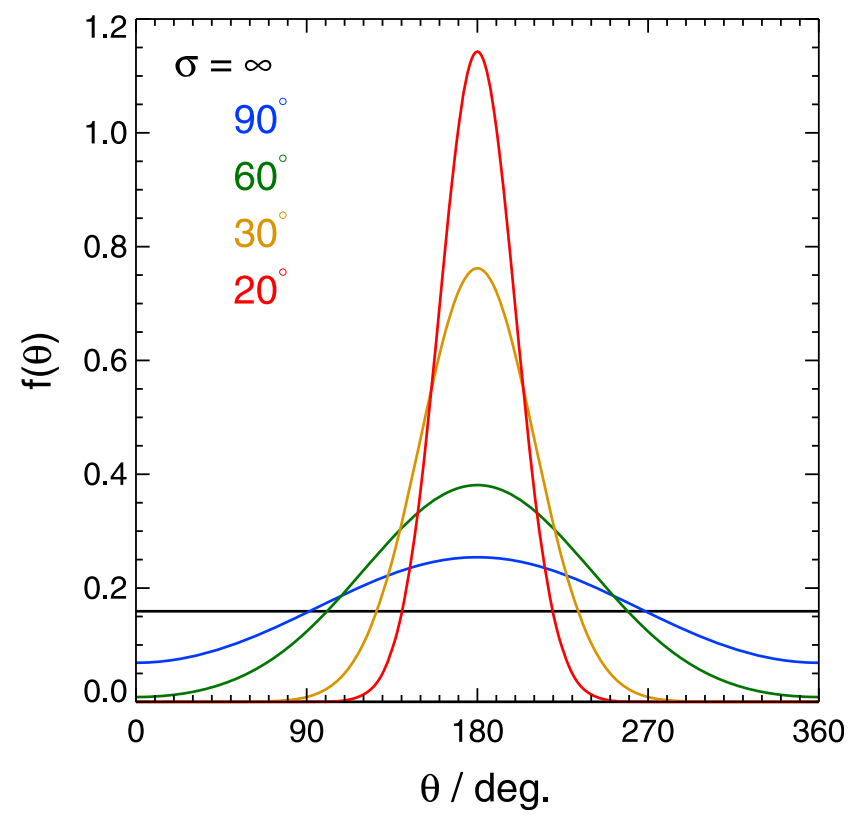

Figure A1. Plots showing the "wrapped normal" distribution $f(\theta)$ given by equation (A3a) computed with a directional mean of $180^{\circ}$ and various color-coded circular standard deviations as indicated in the legend.

[59] Related considerations can also be used for curve fitting. In section 3.3 we wish to find the value of the constant phase angle $\psi^{*}$ such that the function $\psi^{*}-\left\langle\Delta \varphi_{S K R}^{\prime}\right\rangle$ best fits the phase data $\psi_{k}$. In other words we wish to find the value of $\psi^{*}$ such that the quantity $\left.\Delta \psi_{k}=\psi_{k}-\left(\psi^{*}-\left\langle\Delta \varphi_{S K R}^{\prime}\right\rangle\right)\right)$ has the least variance about zero. Following equation $(\mathrm{A} 2 \mathrm{c})$ this variance is given by

$$
\begin{aligned}
V^{\prime} & \left.=\frac{1}{K} \sum_{k=1}^{K}\left(1-\cos \left(\left(\psi_{k}+\left\langle\Delta \varphi_{S K R}^{\prime}\right\rangle\right\rangle\right)-\psi^{*}\right)\right) \\
& =1-\bar{R}^{\prime}+2 \bar{R}^{\prime} \sin ^{2}\left(\overline{\frac{\psi+\Delta \varphi_{S K R}^{\prime}}{2}-\psi^{*}}\right)
\end{aligned}
$$

where

$$
\frac{1}{K} \sum_{k=1}^{K} e^{i\left(\psi_{k}+\left\langle\Delta \varphi_{S K R k}^{\prime}\right\rangle\right)}=\overline{R^{\prime}} e^{\overline{\psi+\Delta \varphi_{S K R}^{\prime}}}
$$

Thus the minimum value of the variance occurs when $\psi^{*}=\overline{\psi+\Delta \varphi_{S K R}^{\prime}}$, i.e., when $\psi^{*}$ is equal to the directional mean of the summed angles $\left(\psi_{k}+\left\langle\Delta \varphi_{S K R}^{\prime}{ }_{k}\right\rangle\right)$. The same methodology is used to fit the difference magnetic phase curve to the data in Figure 8b.

[60] Acknowledgments. Work at Leicester was supported by STFC grant ST/H002480/1. B.C., L.L., and P.Z. were supported by CNES funding to l'Observatoire de Paris; M.K.D. was supported by STFC funding to Imperial College; and D.J.A. was supported by a STFC Quota Studentship. We thank S. Kellock and the Cassini team at Imperial College for access to processed magnetic field data.

[61] Masaki Fujimoto thanks the reviewer for his or her assistance in evaluating this paper.

\section{References}

Andrews, D. J., E. J. Bunce, S. W. H. Cowley, M. K. Dougherty, G. Provan, and D. J. Southwood (2008), Planetary period oscillations in Saturn's magnetosphere: Phase relation of equatorial magnetic field oscillations and SKR modulation, J. Geophys. Res., 113, A09205, doi:10.1029/ 2007JA012937.

Andrews, D. J., S. W. H. Cowley, M. K. Dougherty, and G. Provan (2010a), Magnetic field oscillations near the planetary period in Saturn's equatorial magnetosphere: Variation of amplitude and phase with radial distance and local time, J. Geophys. Res., 115, A04212, doi:10.1029/ 2009JA014729.

Andrews, D. J., A. J. Coates, S. W. H. Cowley, M. K. Dougherty, L. Lamy, G. Provan, and P. Zarka (2010b), Magnetospheric period oscillations at Saturn: Comparison of equatorial and high-latitude magnetic field periods with north and south SKR periods, J. Geophys. Res., 115, A12252, doi:10.1029/2010JA015666.

Arridge, C. S., N. André, N. Achilleos, K. K. Khurana, C. L. Bertucci, L. K. Gilbert, G. R. Lewis, and M. K. Dougherty (2008), Thermal electron periodicities at 20R $\mathrm{R}_{\mathrm{S}}$ in Saturn's magnetosphere, Geophys. Res. Lett., 35, L15107, doi:10.1029/2008GL034132.

Burch, J. L., A. D. DeJong, J. Goldstein, and D. T. Young (2009), Periodicity in Saturn's magnetosphere: Plasma cam, Geophys. Res. Lett., 36, L14203, doi:10.1029/2009GL039043.

Carbary, J. F., and S. M. Krimigis (1982), Charged particle periodicity in the Saturnian magnetosphere, Geophys. Res. Lett., 9, 1073-1076, doi:10.1029/GL009i009p01073.

Carbary, J. F., D. G. Mitchell, S. M. Krimigis, and N. Krupp (2007a), Electron periodicities in Saturn's outher magnetosphere, J. Geophys. Res., 112 A03206, doi:10.1029/2006JA012077

Carbary, J. F., D. G. Mitchell, S. M. Krimigis, and N. Krupp (2007b), Evidence for spiral pattern in Saturn's magnetosphere using the new SKR longitudes, Geophys. Res. Lett., 34, L13105, doi:10.1029/2007GL030167.

Carbary, J. F., D. G. Mitchell, S. M. Krimigis, D. C. Hamilton, and N. Krupp (2007c), Spin-period effects in magnetospheres with no axial tilt, Geophys. Res. Lett., 34, L18107, doi:10.1029/2007GL030483.

Carbary, J. F., D. G. Mitchell, P. Brandt, C. Paranicas, and S. M. Krimigis (2008a), ENA periodicities at Saturn, Geophys. Res. Lett., 35, L07102, doi:10.1029/2008GL033230.

Carbary, J. F., D. G. Mitchell, P. Brandt, E. C. Roelof, and S. M. Krimigis (2008b), Periodic tilting of Saturn's plasma sheet, Geophys. Res. Lett., 35, L24101, doi:10.1029/2008GL036339.

Carbary, J. F., S. M. Krimigis, D. G. Mitchell, C. Paranicas, and P. Brandt (2009a), Energetic neutral atom (ENA) and charged particle periodicities in Saturn's magnetosphere, Adv. Space Res., 44, 483-493, doi:10.1016/ j.asr.2009.04.019.

Carbary, J. F., D. G. Mitchell, S. M. Krimigis, and N. Krupp (2009b), Dual periodicities in energetic electrons at Saturn, Geophys. Res. Lett., 36, L20103, doi:10.1029/2009GL040517.

Carbary, J. F., D. G. Mitchell, S. M. Krimigis, D. A. Gurnett, and W. S. Kurth (2010), Phase relations between energetic neutral atom intensities and kilometric radio emissions at Saturn, J. Geophys. Res., 115, A01203, doi:10.1029/2009JA014519.

Cecconi, B., L. Lamy, P. Zarka, R. Prangé, W. S. Kurth, and P. Louarn (2009), Gonipolarimetric study of the revolution 29 perikrone using the Cassini Radio and Plasma Wave Science instrument high-frequency radio receiver, J. Geophys. Res., 114, A03215, doi:10.1029/2008JA013830.

Clarke, J. T., et al. (2005), Morphological differences between Saturn's ultraviolet aurorae and those of Earth and Jupiter, Nature, 433, 717-719, doi:10.1038/nature03331.

Clarke, K. E., et al. (2006), Cassini observations of planetary-period oscillations of Saturn's magnetopause, Geophys. Res. Lett., 33, L23104, doi:10.1029/2006GL027821.

Clarke, K. E., D. J. Andrews, A. J. Coates, and S. W. H. Cowley (2010a), Magnetopause oscillations near the planetary period at Saturn: Occurrence, phase and amplitude, J. Geophys. Res., 115, A08209, doi:10.1029/ 2009JA014745.

Clarke, K. E., D. J. Andrews, A. J. Coates, S. W. H. Cowley, and A. Masters (2010b), Magnetospheric period oscillations of Saturn's bow shock, J. Geophys. Res., 115, A05202, doi:10.1029/2009JA015164.

Connerney, J. E. P., N. F. Ness, and M. H. Acuña (1982), Zonal harmonic model of Saturn's magnetic field from Voyager 1 and 2 observations, Nature, 298, 44-46, doi:10.1038/298044a0.

Cowley, S. W. H., D. M. Wright, E. J. Bunce, A. C. Carter, M. K. Dougherty, G. Giampieri, J. D. Nichols, and T. R. Robinson (2006), Cassini observations of planetary- period magnetic field oscillations in Saturn's magnetosphere: Doppler shifts and phase motion, Geophys. Res. Lett., 33, L07104, doi:10.1029/2005GL025522. 
Desch, M. D., and M. L. Kaiser (1981), Voyager measurement of the rotation period of Saturn's magnetic field, Geophys. Res. Lett., 8, 253-256, doi:10.1029/GL008i003p00253.

Dougherty, M. K., et al. (2004), The Cassini magnetic field investigation, Space Sci. Rev., 114, 331-383, doi:10.1007/s11214-004-1432-2.

Dougherty, M. K., et al. (2005), Cassini magnetometer observations during Saturn orbit insertion, Science, 307, 1266-1270, doi:10.1126/ science. 1106098.

Espinosa, S. A., and M. K. Dougherty (2000), Periodic perturbations in Saturn's magnetic field, Geophys. Res. Lett., 27, 2785-2788, doi:10.1029/2000GL000048.

Espinosa, S. A., D. J. Southwood, and M. K. Dougherty (2003a), Reanalysis of Saturn's magnetospheric field data in view of spin-periodic perturbations, J. Geophys. Res., 108(A2), 1085, doi:10.1029/2001JA005083.

Espinosa, S. A., D. J. Southwood, and M. K. Dougherty (2003b), How can Saturn impose its rotation period in a noncorotating magnetosphere? J. Geophys. Res., 108(A2), 1086, doi:10.1029/2001JA005084.

Farrell, W. M., M. D. Desch, M. L. Kaiser, A. Lecacheux, W. S. Kurth, D. A. Gurnett, B. Cecconi, and P. Zarka (2005), A nightside source of Saturn kilometric radiation: Evidence for an inner magnetosphere energy driver, Geophys. Res. Lett., 32, L18107, doi:10.1029/2005GL023449.

Galopeau, P. H. M., and A. Lecacheux (2000), Variations of Saturn's radio rotation period measured at kilometer wavelengths, J. Geophys. Res. 105, 13,089-13,101, doi:10.1029/1999JA005089.

Galopeau, P. H. M., P. Zarka, and D. Le Quéau (1995), Source location of Saturn's kilometric radiation: The Kelvin-Helmholtz instability hypothesis, J. Geophys. Res., 100, 26,397-26,410, doi:10.1029/95JE02132.

Gérard, J.-C., et al. (2006), Saturn's auroral morphology and activity during quiet magnetospheric conditions, J. Geophys. Res., 111, A12210, doi:10.1029/2006JA011965.

Giampieri, G., M. K. Dougherty, E. J. Smith, and C. T. Russell (2006), A regular rotation period for Saturn's magnetic field that may track its internal rotation, Nature, 441, 62-64, doi:10.1038/nature04750.

Grodent, D., J.-C. Gérard, S. W. H. Cowley, E. J. Bunce, and J. T. Clarke (2005), Variable morphology of Saturn's southern ultraviolet aurora, J. Geophys. Res., 110, A07215, doi:10.1029/2004JA010983.

Gurnett, D. A., W. S. Kurth, and F. L. Scarf (1981), Plasma waves near Saturn: Initial results from Voyager 1, Science, 212, 235-239, doi:10.1126/ science.212.4491.235

Gurnett, D. A., et al. (2004), The Cassini radio and plasma wave investigation, Space Sci. Rev., 114, 395-463, doi:10.1007/s11214-004-1434-0.

Gurnett, D. A., et al. (2005), Radio and plasma wave observations at Saturn from Cassini's approach and first orbit, Science, 307, 1255-1259, doi:10.1126/science. 1105356 .

Gurnett, D. A., A. M. Persoon, W. S. Kurth, J. B. Groene, T. F. Averkamp, M. K. Dougherty, and D. J. Southwood (2007), The variable rotation period of the inner region of Saturn's plasma disk, Science, 316 , 442-445, doi:10.1126/science. 1138562.

Gurnett, D. A., A. Lecacheux, W. S. Kurth, A. M. Persoon, J. B Groene, L. Lamy, P. Zarka, and J. F. Carbary (2009a), Discovery of a north-south asymmetry in Saturn's radio rotation period, Geophys. Res. Lett., 36, L16102, doi:10.1029/2009GL039621.

Gurnett, D. A., A. M. Persoon, J. B. Groene, A. J. Kopf, G. B. Hospodarsky, and W. S. Kurth (2009b), A north-south difference in the rotation rate of auroral hiss at Saturn: Comparison to Saturn's kilometric radio emission, Geophys. Res. Lett., 36, L21108, doi:10.1029/2009GL040774.

Gurnett, D. A., et al. (2010a), A plasmapause-like density boundary at high latitudes in Saturn's magnetosphere, Geophys. Res. Lett., 37, L16806, doi:10.1029/2010GL044466.

Gurnett, D. A., J. B. Groene, A. M. Persoon, J. D. Menietti, S.-Y. Ye, W. S. Kurth, R. J. MacDowell, and A. Lecacheux (2010b), The reversal of the rotational modulation rates of the north and south components of Saturn kilometric radiation near equinox, Geophys. Res. Lett., 37, L24101, doi:10.1029/2010GL045796.

Kaiser, M. L., and M. D. Desch (1982), Saturn kilometric radiation: Source locations, J. Geophys. Res., 87, 4555-4559, doi:10.1029/ JA087iA06p04555.

Kaiser, M. L., M. D. Desch, J. W. Warwick, and J. B. Pearce (1980) Voyager detection of non-thermal radio emission from Saturn, Science, 209, 1238-1240, doi:10.1126/science.209.4462.1238.

Kaiser, M. L., M. D. Desch, and A. Lecacheux (1981), Saturn kilometric radiation: Statistical properties and beam geometry, Nature, 292, 731-733, doi:10.1038/292731a0.

Khurana, K. K., D. G. Mitchell, C. S. Arridge, M. K. Dougherty, C. T. Russell, C. Paranicas, N. Krupp, and A. J. Coates (2009), Sources of rotational signals in Saturn's magnetosphere, J. Geophys. Res., 114, A02211, doi:10.1029/2008JA013312.
Krupp, N., et al. (2005), The Saturnian plasma sheet as revealed by energetic particle measurements, Geophys. Res. Lett., 32, L20S03, doi:10.1029/ 2005GL022829.

Kurth, W. S., A. Lecacheux, T. F. Averkamp, J. B. Groene, and D. A Gurnett (2007), A Saturnian longitude system based on a variable kilometric radiation period, Geophys. Res. Lett., 34, L02201, doi:10.1029/ 2006GL028336.

Kurth, W. S., T. F. Averkamp, D. A. Gurnett, J. B. Groene, and A. Lecacheux (2008), An update to a Saturnian longitude system based on kilometric radio emissions, J. Geophys. Res., 113, A05222, doi:10.1029/ 2007JA012861.

Lamy, L. (2011), Variability of southern and northern SKR periodicities, in Proceedings of the Seventh International Workshop on Planetary, Solar and Heliospheric Radio Emissions, edited by H. O. Rucker et al., Austrian Acad. Sci., in press.

Lamy, L., P. Zarka, B. Cecconi, R. Prangé, W. S. Kurth, and D. A. Gurnett (2008a), Saturn kilometric radiation: Average and statistical properties, J. Geophys. Res., 113, A07201, doi:10.1029/2007JA012900.

Lamy, L., P. Zarka, B. Cecconi, S. Hess, and R. Prangé (2008b), Modeling of Saturn kilometric radiation arcs and equatorial shadow zone, J. Geophys. Res., 113, A10213, doi:10.1029/2008JA013464.

Lamy, L., B. Cecconi, R. Prangé, P. Zarka, J. D. Nichols, and J. T. Clarke (2009), An auroral oval at the footprint of Saturn's kilometric radio sources, co-located with the UV aurorae, J. Geophys. Res., 114, A10212, doi:10.1029/2009JA014401.

Lamy, L., et al. (2010), Properties of Saturn kilometric radiation measured within its source region, Geophys. Res. Lett., 37, L12104, doi:10.1029/ 2010GL043415

Lecacheux, A., and F. Genova (1983), Source localization of Saturn kilometric radio emission, J. Geophys. Res., 88, 8993-8998, doi:10.1029/ JA088iA11p08993.

Mardia, K. V., and P. E. Jupp (2000), Directional Statistics, Wiley, Chichester, U. K.

Mutel, R. L., J. D. Menietti, D. A. Gurnett, W. Kurth, P. Schippers, C. Lynch, L. Lamy, C. S. Arridge, and B. Cecconi (2010), CMI growth rates for Saturnian kilometric radiation, Geophys. Res. Lett., 37, L19105, doi:10.1029/2010GL044940.

Nichols, J. D., J. T. Clarke, S. W. H. Cowley, J. Duval, A. J. Farmer, J.-C. Gérard, D. Grodent, and S. Wannawichian (2008), Oscillation of Saturn's southern auroral oval, J. Geophys. Res., 113, A11205, doi:10.1029/ 2008JA013444.

Nichols, J. D., B. Cecconi, J. T. Clarke, S. W. H. Cowley, J.-C. Gérard, A. Grocott, D. Grodent, L. Lamy, and P. Zarka (2010a), Variation of Saturn's UV aurora with SKR phase, Geophys. Res. Lett., 37, L15102, doi:10.1029/2010GL044057.

Nichols, J. D., S. W. H. Cowley, and L. Lamy (2010b), Dawn-dusk oscillation of Saturn's conjugate auroral ovals, Geophys. Res. Lett., 37, L24102, doi:10.1029/2010GL045818.

Provan, G., D. J. Andrews, C. S. Arridge, S. W. H. Cowley, S. E. Milan, M. K. Dougherty, and D. M. Wright (2009a), Polarization and phase of planetary period oscillations on high latitude field lines in Saturn's magnetosphere, J. Geophys. Res., 114, A02225, doi:10.1029/2008JA013782.

Provan, G., S. W. H. Cowley, and J. D. Nichols (2009b), Phase relation of oscillations near the planetary period of Saturn's auroral oval and the equatorial magnetospheric magnetic field, J. Geophys. Res., 114 A04205, doi:10.1029/2008JA013988.

Provan, G., D. J. Andrews, B. Cecconi, S. W. H. Cowley, M. K. Dougherty, L. Lamy, and P. Zarka (2011), Magnetospheric period magnetic field oscillations at Saturn: Equatorial phase "jitter" produced by superposition of southern- and northern-period oscillations, J. Geophys. Res., 116 , A04225, doi:10.1029/2010JA016213.

Smith, E. J., L. Davis Jr., D. E. Jones, P. J. Coleman Jr., D. S. Colburn, P. Dyal, and C. P. Sonett (1980), Saturn's magnetic field and magnetosphere, Science, 207, 407-410, doi:10.1126/science.207.4429.407.

Southwood, D. J. (2011), Direct evidence of differences in magnetic rotation rate between Saturn's northern and southern polar regions, J. Geophys. Res., 116, A01201, doi:10.1029/2010JA016070.

Southwood, D. J., and M. G. Kivelson (2007), Saturn magnetospheric dynamics: Elucidation of a camshaft model, J. Geophys. Res., 112 A12222, doi:10.1029/2007JA012254

Southwood, D. J., and M. G. Kivelson (2009), The source of Saturn's periodic radio emission, J. Geophys. Res., 114, A09201, doi:10.1029/ 2008JA013800

Trauger, J. T., et al. (1998), Saturn's hydrogen aurora: Wide field and planetary camera 2 imaging from the Hubble Space Telescope, J. Geophys. Res., 103, 20,237-20,244, doi:10.1029/98JE01324

Wang, Z., D. A. Gurnett, G. Fischer, S.-Y. Ye, W. S. Kurth, D. G. Mitchell, J. S. Leisner, and C. T. Russell (2010), Cassini observations of narrow- 
band radio emissions in Saturn's magnetosphere, J. Geophys. Res., 115, A06213, doi:10.1029/2009JA014847.

Warwick, J. W., et al. (1981), Planetary radio astronomy observations from Voyager-1 near Saturn, Science, 212, 239-243, doi:10.1126/science. 212.4491.239.

Warwick, J. W., D. S. Evans, J. H. Romig, J. K. Alexander, M. D. Desch, M. L. Kaiser, M. Aubier, Y. Leblanc, A. Lecacheux, and B. M. Pedersen (1982), Planetary radio astronomy observations from Voyager-2 near Saturn, Science, 215, 582-587, doi:10.1126/science.215.4532.582.

$\mathrm{Wu}, \mathrm{C}$. S., and L. C. Lee (1979), A theory of the terrestrial kilometric radiation, Astrophys. J., 230, 621-626, doi:10.1086/157120.

Ye, S.-Y., D. A. Gurnett, J. B. Groene, Z. Wang, and W. S. Kurth (2010) Dual periodicities in the rotational modulation of Saturn narrowband emissions, J. Geophys. Res., 115, A12258, doi:10.1029/2010JA015780.
Zarka, P. (1998), Auroral radio emissions at the outer planets: Observations and theories, J. Geophys. Res., 103, 20,159-20,194, doi:10.1029/ 98JE01323.

Zarka, P., L. Lamy, B. Cecconi, R. Prangé, and H. O. Rucker (2007), Modulation of Saturn's radio clock by solar wind speed, Nature, 450, 265-267, doi:10.1038/nature06237.

D. J. Andrews (corresponding author), S. W. H. Cowley, and G. Provan, Department of Physics and Astronomy, University of Leicester, University Road, Leicester LE1 7RH, UK. (dja13@ion.le.ac.uk)

B. Cecconi, L. Lamy, and P. Zarka, LESIA, Observatoire de Paris, CNRS, UPMC, Université Paris Diderot, 5 place J. Janssen, Meudon F-92190, France.

M. K. Dougherty, Blackett Laboratory, Imperial College, Exhibition Road, London SW7 2AZ, UK. 